\title{
Petri nets conflicts resolution for performance evaluation and control of urban bus networks: a $(\max ,+)$ based approach
}

\author{
Y. Idel Mahjoub ${ }^{\mathrm{a}, \mathrm{b}}$, A. Nait-Sidi-Moh ${ }^{\mathrm{a}}$, E. Chakir El Alaoui ${ }^{\mathrm{b}}$ and A. Tajer ${ }^{\mathrm{c}}$ \\ ${ }^{a}$ LTI laboratory, University of Picardie Jules Verne-INSSET, 02100 Saint-Quentin, France; \\ ${ }^{b}$ LSET laboratory, Cadi Ayyad University-FSTG, 40000, Marrakesh, Morocco; \\ ${ }^{c}$ LGECOS laboratory, Cadi Ayyad University-ENSA, 40000, Marrakesh, Morocco
}

\section{ARTICLE HISTORY}

Compiled September 20, 2018

\begin{abstract}
This paper deals with the modeling, analysis, performance evaluation and control of urban bus networks characterized by conflicts, synchronization and concurrency using two complementary formalism Petri nets (PN) and dioid algebra. We mainly focused on conflicts and resource sharing resolution using some routing functions in order to evaluate some passengers metrics within various stations of the bus network. More precisely, we evaluate arrival, boarding, disembarking and waiting times of each passenger at any network station. As a second contribution of this paper, we study through the developed models the influence of the limited capacity of the buses on passengers travel time. The objective is to find an optimal threshold from which the buses capacity has no longer impact on passengers waiting time. Finally, we propose a control approach enabling to determine a compromise between the capacity and number of buses to use on the network in order to minimize both waiting time of passengers and transportation company costs by using buses with suitable capacities especially in peak periods. An illustrative example is given to show the applicability of the proposed approach and the obtained results are promising.
\end{abstract}

\section{KEYWORDS}

Transportation systems; Modeling; Performance evaluation; Petri Nets; Dioid

Algebra; Conflicts; Resource sharing; Control

\section{Introduction}

The problems of congestion, pollution (atmospheric and sound) and road insecurity, encourage public authorities and transport companies to increase efforts to maintain and improve the quality of services offered to users, to make the collective ways of transportation more attractive (Nait-Sidi-Moh, Manier, and Moudni (2009)). Thus, the study and analysis of such systems have become one of the major concerns of researchers in the field of public transport, in order to propose improvements that can ensure the development of such networks and contribute to the efficiency of various services proposed to users (Nait-Sidi-Moh, Manier, and Moudni (2003)).

Transportation networks can be seen as a Discrete Event System (DES). These systems are characterized by discrete events and states (such as state of buses: moving or stopping, state of passengers: waiting or transported). Changes of system states may be 
involved by the occurrence of some discrete events and conducts the system to another state. Among these events, we underline arrival/departure of a bus to/from a network station, a passenger getting on/off the bus. The behavior of such systems is mainly gouverned by specific functioning integrating some phenomena like synchronization, concurrency and conflicts explained later in this section. In order to model, analyze and solve these complex phenomena, many tools and methods have been developed in the literature. Although, simulation based approaches and operational programming are still widely used to evaluate DES performances, there is increasing demand for a development of mathematical formulations that produce suitable solutions to these complex phenomena. Petri nets combined with dioid algebra are two complementary formalisms that fulfill this aim by both providing a clear graphical models for analysis and simulation of the studied system and also a useful mathematical equations that describes the dynamical evolution of the system. Furthermore, dioid algebra is one of the famous linear and stationary mathematical tools developed for DES nevertheless it is not sufficiently developed to support DES with conflicts and resource sharing. In this work, the term of conflict refers to situations wherein it is needed to take a decision and make a choice between many possibilities.

To overcome this lack, our study focuses on the development of $(\max ,+)$ models capable to represent the problems of conflicts, concurrency and bringing solutions for performance evaluation and analysis of transportation networks as a sub class of DES. Actually, we first represent the behavior of the system with a PN model, and then translate easily the system behavior into $(\max ,+)$ equations. In fact, $\mathrm{PN}$ are used thanks to their powerful formalism, their conceptual simplicity and intuitive graphical presentation for modeling DES. Moreover, using a PN model, it is easier to describe the analytic behavior of the studied system with $(\max ,+)$ algebra. In our study, this exotic algebra is used thanks to its powerful mathematical basis that allows a linear analytic description of DES. Our ultimate objective, is to use this algebra to handle and analyze the conflict aspect of the considered system.

The studied system, in this paper, is a bus network composed of two lines connected by a common station (connection station). Each bus line is composed of a finite number of stations and served by a finite number of buses with limited capacities. This network involves several different choices (such as a bus making a correspondence or not within a connection stop, a passenger getting on/off a bus or not, passengers with different origins and destinations). This is expressed formally in the equation (13) where a conflict situation is observed between the transitions $x_{1, j}^{i}, . ., x_{p_{i}, j}^{i}$ as output transitions of the place $P b_{j}^{i}$ (see figure 4). Other phenomena are observed in our studied system such as synchronization (i.e. meaning synchronized arrival and departure of buses at a given station, which is expressed formally by the equation (1)), and concurrency (i.e. boarding/disembarking of passengers with limited resources in term of buses and their capacities, which is expressed formally by the equation (6) and (14)). Further details on these phenomena can be found in Baccelli et al. (1992), Gaubert (1999) and Boussahel, Amari, and Kara (2016).

In this paper, we present a modeling approach based on linear and non-stationary $(\max ,+)$ equations obtained from a PN model describing the behavior of a bus transportation network. The originality of this paper lies firstly in the use of $(\max ,+)$ algebra to describe the dynamic behavior of Petri net-based models with structural and behavioral conflicts, and secondly in the combination of the advantages of these formal formalisms and their efficiency for the modeling and evaluation of the performances of such DES. In fact, the presence of conflicts conducts to a non-stationary $(\max ,+)$ model that leads us to express and solve some functions called routing func- 
tions to manage and arbitrate the encountered conflicts. Afterwards, some quantitative properties of the system are evaluated based on obtained models. We mainly focused on arrival and departure times of buses at different stations, boarding, disembarking and waiting times of each passenger at every station. Furthermore, the influence of limited capacity of buses on passengers travel time is studied in order to find an optimal capacity from which this capacity has no longer impact on passengers travel time. The developed models present four main advantages. First, these models consider more constraints related namely to buses finite capacity, random passengers arrival and the choice of their destination. Second, this approach is extended for networks of huge size with several lines connected by correspondence stops. Third, the particularity and the strength of the developed models lie in their ability to be applied to any other public transport network (such as railway networks). Finally, the proposed models can be considered as a decision making system for transportation companies in order to minimize their costs by using buses with suitable capacities especially in peak periods. This will considerably participate to minimize both the number of used buses on the network and the waiting times of passengers.

The remainder of this paper is organized as follows. In section 2, the related work is presented. The considered public transport system is introduced in section 3 . In Section 4, PN model describing the graphical behaviour of the considered network is proposed. A mathematical modeling using $(\max ,+)$ algebra is given in section 5 . Performance evaluation and adopted control policies are presented in section 6 . An illustrative numerical example is presented in section 7 . The last section concludes the paper and presents some perspectives.

\section{Related work}

Public transportation networks are subject to complex phenomena which make their study more difficult and require the use of adequate tools. In fact, this difficulty is increased by the complexity of the studied system due for example to the diversity of the parameters to be taken into account during the modeling, to the variable conditions of the traffic and to the random aspect of the transport demand. Among these tools used for the study of public transport systems, we underline Petri nets (Diaz (2001)), multi-agent systems (Capkovic (2016), Adler and Blue (2002)), multicriteria analysis (Yeh, Deng, and Chang (2000)), dioid algebra (Gaubert (1999), Houssin, Lahaye, and Boimond (2013)), Markov decision process (Nuzzolo and Comi (2017)) etc, allowing to describe the behavior of such systems in a formal way. For instance, some research focuses on methods which can be used in real time to estimate and forecast transit performance, especially vehicles occupancy and crowding (Nuzzolo and Comi (2016)). For example, in order to monitor the number of passengers relative to each transit vehicle, several passenger count technologies are applied (Nielsen et al. (2014)). These data can be used to estimate in real time and to forecast in the short term the number of passengers waiting at various stations and on board, which is used to estimate the occupancy rate and crowding. Other research focuses on multi-criteria models (Borges et al. (2016)) that provide support to managers and directors of transport systems, offering a comprehensive view that supports the implementation of public policies for the improvement of transport services. Later on, we focus on methods and models, based on PN and/or dioid algebra, used for performance evaluation and control of DES including transportation networks. The objective is to point out the lack of current literature that we would like to overcome by proposing some original models and also 
to allow reader to position our contribution in the literature.

Several researchers have been interested in studying various kinds of DES, including transportation networks, based on Petri nets and dioid algebra (Gaubert (1999), NaitSidi-Moh, Manier, and Moudni (2003), Nait-Sidi-Moh, Manier, and Moudni (2009), Benarbia et al. (2012), Heidergott and de Vries (2001), Idel Mahjoub, Chakir El Alaoui, and Nait-Sidi-Moh (2017), Houssin, Lahaye, and Boimond (2006)). Previous research is divided into two fields: modeling and evaluation of performance on the one hand and regulation problems on the other hand.

First of all, the main use of Petri nets and/or dioid algebra in the field of transportation systems have focused on modeling, analysis and evaluation of performances. For instance, Bouyekhf et al. (2003) proposed an approach to schedule the public transport hub-based network using generalized stochastic Petri nets. This approach in the public transport field allows to improve the timetables in term of connection costs in order to minimize passenger waiting times. Moreover, spectral theory in $(\max ,+)$ algebra has been used, in Nait-Sidi-Moh, Manier, and Moudni (2009), for evaluation of passengers waiting times at the connection stations within a bus network. Furthermore, Benarbia et al. (2012) used stochastic PN for modeling and analysing of the performances of self-service bicycles. This study focused on rebalancing the distribution of bicycles in various network stations in order to satisfy demands of users. Similarly, Labadi et al. (2015) developed a discrete event approach for modeling and performance evaluation of public bicycle-sharing systems using timed PN with inhibitor arcs and variable arc weights. Lahaye, Houssin, and Boimond (2003) modeled the behavior of a urban bus network by a min-max recursive equation which can be used for the simulation issue in real time.

On the other hand, several studies have been considered for regulation problem in transportation networks (Bonhomme (2013), Heidergott and de Vries (2001)). One of the addressed issues in these studies is to find a new schedule of multiple vehicles after the detection of a disturbance on the road at a given time. For example, Hakim (2002) proposed a multi-agent system for regulating the correspondence in real time. Furthermore, a genetic approach for scheduling a transportation network was proposed in Dridi (2004). In addition, Ould-Sidi et al. (2006) proposed a fuzzy evolutionary approach for regulation of an urban transport network in order to facilitate decision making for regulators in real time. Also, Cacchiani et al. (2014) proposed an overview of repair models and algorithms for real-time railway disturbance and disruption management, in order to increase the quality of the provided railway services. In the same field, Kersbergen et al. (2016) presented how to model railway traffic and dispatching actions like changing tracks and breaking or joining trains using the max-plus algebra. A model predictive controller was proposed in order to manage the railway traffic in real time. Furthermore, given that the dynamic behavior of traffic lights regulating a network of intersections can be viewed as a complex discrete-event system that can be modeled and controlled by Petri nets, List and Mashayekhi (2015) presented a modular colored stochastic PN for modeling and analysis of signalized intersections. This study has significant promise, especially in light of the increasing demands for more features and also real-time control.

Comparing the presented approach with other models based on PN and $(\max ,+)$ algebra (e.g, Nait-Sidi-Moh, Manier, and Moudni (2009), Lahaye, Houssin, and Boimond (2003)), it may be enlightened that our contribution does not lie only in the modeling of the dynamics associated with the buses circuits and their timetables, but also in the evaluation of passengers random arrivals, their boarding and disembarking times as well as their differentiated itineraries and destinations. In addition, some 
models in the literature such as Houssin, Lahaye, and Boimond (2013) consider a control approach for timetables synthesis using $(\max ,+)$ algebra without taking into account buses finite capacities. However, in this paper, a control approach enabling to determine a compromise between the capacity and number of buses to use on the network is performed in order to minimize both waiting times of passengers and transportation company costs. Furthermore, the proposed approach could also be applied to any other public transportation system (railway,...). The developed models are validated on several scenarios and configurations of the system, and the obtained results are satisfying and promising. This paper extends and generalizes the work presented in Idel Mahjoub, Chakir El Alaoui, and Nait-Sidi-Moh (2017) while considering any number of stations for each bus line instead of just three. In fact, all the equations and algorithms obtained in the initial work are now extended and generalized.

\section{Studied system}

In this section, we consider a bus network composed of two connected lines $\mathrm{L}_{i}$ and $\mathrm{L}_{i+1}$ with a single connection station (figure 1). This system configuration can be considered to be a generic structure for any bus transportation network. Each line $\mathrm{L}_{l}(\mathrm{l} \in\{\mathrm{i}, \mathrm{i}+1\})$ is represented by $\mathrm{p}_{l}$ stops : departure stop $\left(\mathrm{S}_{1}^{l}\right)$, connection stop $\left(\mathrm{S}_{q_{l}}^{l}\right)$, intermediate stops $\left(\mathrm{S}_{2}^{l}, \ldots, \mathrm{S}_{q_{l}-1}^{l}, \mathrm{~S}_{q_{l}+1}^{l}, \ldots, \mathrm{S}_{p_{l}-1}^{l}\right)$, and an arrival terminus $\left(\mathrm{S}_{p_{l}}^{l}\right)$. Each line $\mathrm{L}_{l}$ is supposed to be served by a finite number $\mathrm{n}_{l}$ of buses and that each bus $\mathrm{B}_{j}^{l}$ is characterized by its capacity $\mathrm{C}_{j}^{l}$ (figure 1$)\left(\mathrm{C}_{j}^{l}\right.$ is the maximum number of passengers that can board the bus $\mathrm{B}_{j}^{l}$ ). The passengers arrive to their departure stops in a random way (stochastic law). For each line $\mathrm{L}_{l}$, the following data are supposed to be known and fixed (for a given period):

- The number $\mathrm{n}_{l}$ of buses circulating on the line $\mathrm{L}_{l}$;

- The travel times $\left(\tau_{2}^{l}, \tau_{4}^{l}, \ldots, \tau_{2 p_{l}-2}^{l}\right)$ of buses between all network stops, where $\tau_{2}^{l}$ (resp. $\tau_{4}^{l}, \ldots, \tau_{2 p_{l}-2}^{l}$ ) is the travel time between $\mathrm{S}_{1}^{l}$ (resp. $\mathrm{S}_{2}^{l}, \ldots, \mathrm{S}_{p_{l}-1}^{l}$ ) and $\mathrm{S}_{2}^{l}$ (resp. $\left.\mathrm{S}_{3}^{l}, \ldots, \mathrm{S}_{p_{l}}^{l}\right)$.

- The stop times $\left(\tau_{1}^{l}, \tau_{3}^{l}, \ldots, \tau_{2 p_{l}-1}^{l}\right)$ of each bus in different stops, where $\tau_{1}^{l}$ (resp. $\tau_{3}^{l}, \ldots, \tau_{2 p_{l}-1}^{l}$ ) is the stop time of the buses at $\mathrm{S}_{1}^{l}$ (resp. $\mathrm{S}_{2}^{l}, . ., \mathrm{S}_{p_{l}}^{l}$ ). These stop times include the mean time needed for a passenger to get on and/or get off the bus denoted $\delta$.

We assume that the destination of each passenger is known and randomly chosen. It means that an arbitrarily possible destination is assigned to each passenger. Considering this destination, we can evaluate boarding, disembarking and travel time of each passenger.

In the following section, we proceed step by step to conceive the PN model of the studied system. The global PN model that represents our system is given in figure 5 .

\section{Modeling using Petri Nets}

$\mathrm{PN}$, namely p-timed $\mathrm{PN}$, stochastic $\mathrm{PN}$ and free choice $\mathrm{PN}$, have been proven to be a powerful modeling formalism for various kinds of DES. In fact, this formalism is useful in verification, validation and performance evaluation. In this section, these 


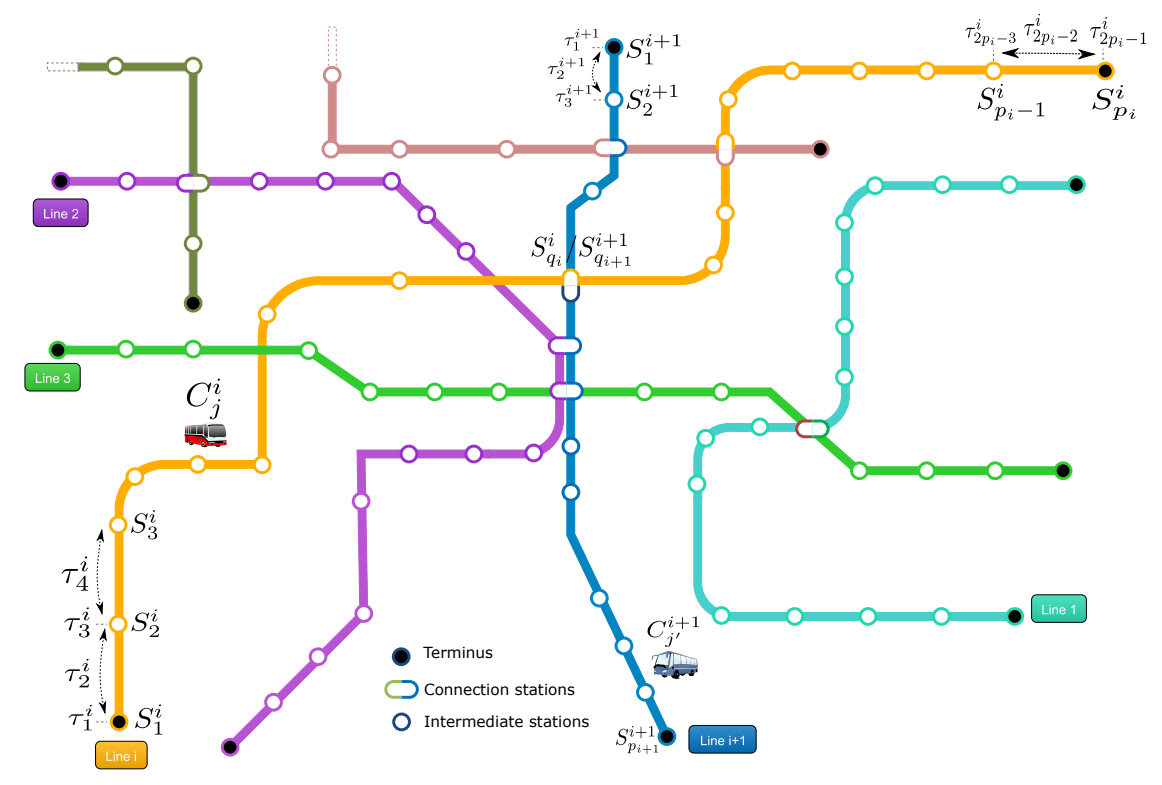

Figure 1. The studied network

sub-classes of PN are used to model respectively (i) bus circuit, (ii) passengers arrival and boarding and finally (iii) passengers destination choice and disembarking, in order to conceive the model of the global network of figure 1. Basic concepts and further details on PN can be found for example in Murata (1989), Baccelli, Foss, and Gaujal (1996), Diaz (2001) and Reisig and Rozenberg (1998). For the remainder, we will only develop models of the line $\mathrm{L}_{i}$. For the other line $\left(\mathrm{L}_{i+1}\right)$, we obtain the same models while replacing the index $i$ (resp. j) by the index $i+1$ (resp. $\left.j^{\prime}\right)$.

\section{1. $\quad$ Modeling bus circuit using PTPN}

The following figure describes the PN model of the circuit of a bus $\mathrm{B}_{j}^{i}$ between the various stations of the line $\mathrm{L}_{i}$ (figure 1 ).

Notation: In this paper, the indexes $\mathrm{i}, \mathrm{j}$ and $\mathrm{s}$ in each notation $\mathrm{X}_{s, j}^{i}$ are given by: $\mathrm{i}$ refers to the bus line $\mathrm{L}_{i}, \mathrm{j}$ refers to the bus $\mathrm{B}_{j}^{i}$ (for line $\mathrm{L}_{i}, 1 \leq \mathrm{j} \leq \mathrm{n}_{i}$ ) and s refers to the station $\mathrm{S}_{s}^{i}$ (for line $\mathrm{L}_{i}, 1 \leq \mathrm{s} \leq \mathrm{p}_{i}$ ).

Each bus $\mathrm{j}$ is initially in its starting station $\mathrm{S}_{1}^{i}$ (presence of a token in the place $\mathrm{Pc}_{j}^{i}$ ). The firing of the transition $\mathrm{u}_{j}^{i}$ represents a departure order given to the bus $\mathrm{j}$. The firing of the transition $\operatorname{Dpt}_{j}^{i}$ means that the bus $\mathrm{j}$ begins its circuit. This bus will wait $\tau_{1}^{i}$ time units before leaving the departure station, so that waiting passengers can get on the bus and $\delta$ refers to the average time that a person can takes to get on/off the bus. $\tau_{2}^{i}$ units of time later, the bus arrives to the second station. The firing of $\mathrm{xa}_{2, j}^{i}$ puts simultaneously a token in the place $\mathrm{P}_{2, j}^{i}$ (which models bus waiting), and another token in the place $\mathrm{Pcm}_{2, j}^{i}$ (which models the authorization order for passengers to get on the bus $\mathrm{j}$ ) and finally another token in the place $\operatorname{Pcd}_{2, j}^{i}$ (which models the authorization order for passengers to get off the bus $j$ ). The same operating mode is 


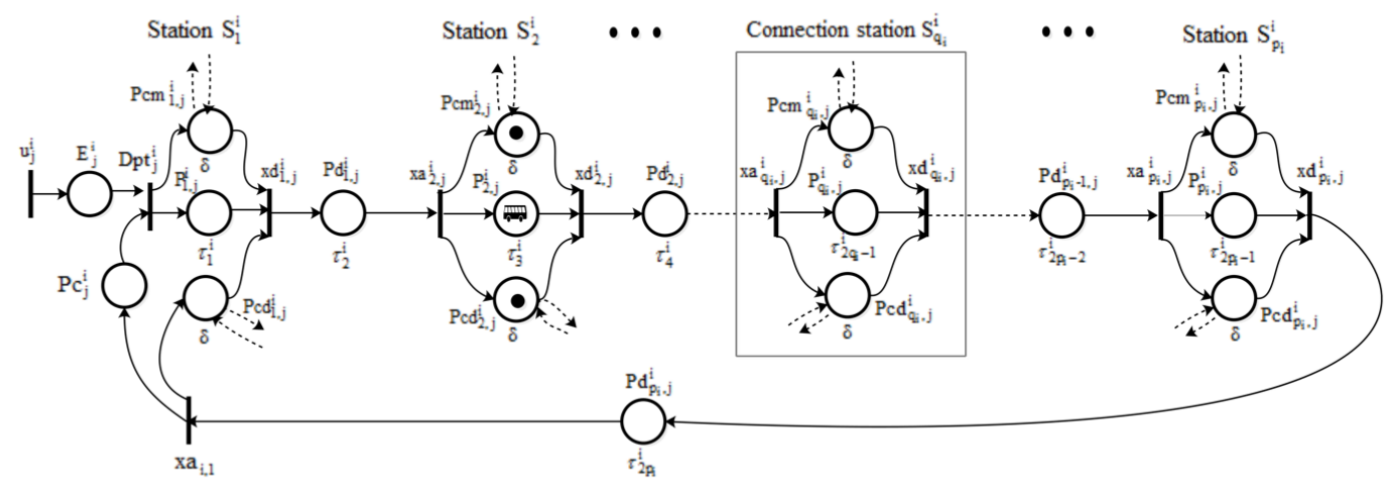

Figure 2. PN model of a bus circuit

Table 1. Elements of PN model of a bus circuit

For $\mathrm{i}, \mathrm{j}$ and $\mathrm{s}\left(1 \leq \mathrm{i}, 1 \leq \mathrm{j} \leq \mathrm{n}_{i}, 1 \leq \mathrm{s} \leq \mathrm{p}_{i}\right)$

\begin{tabular}{l||l}
\multicolumn{1}{l}{ Transitions } \\
\hline $\mathrm{u}_{j}^{i}$ & Departure permission for bus $\mathrm{j}$ \\
\hline $\mathrm{Dpt}_{j}^{i}$ & Travel beginning for bus $\mathrm{j} ;$ \\
\hline $\mathrm{xd}_{s, j}^{i}$ & Departure of bus $\mathrm{j}$ from the station $\mathrm{s} ;$ \\
\hline $\mathrm{xa}_{s, j}^{i}$ & The arrival of bus $\mathrm{j}$ to the station $\mathrm{s} ;$ \\
\hline $\mathrm{Places}$ & \multicolumn{2}{|l|}{ Bus $\mathrm{j}$ ready to begin its circuit; } \\
\hline $\mathrm{E}_{j}^{i}$ & The waiting of bus $\mathrm{j}$ at the station $\mathrm{s} ;$ \\
\hline $\mathrm{P}_{s, j}^{i}$ & Movement of bus $\mathrm{j}$ between station $\mathrm{s}$ and $\mathrm{s}+1 ;$ \\
\hline $\mathrm{Pd}_{s, j}^{i}$ & Bus waiting at its departure station; \\
\hline $\mathrm{Pc}_{j}^{i}$ & Authorization for passengers to get on the bus $\mathrm{j} ;$ \\
\hline $\mathrm{Pcm}_{s, j}^{i}$ & Authorization for passengers to get off the bus $\mathrm{j} ;$ \\
\hline $\mathrm{Pcd}_{s, j}^{i}$ & Temporizations \\
\hline$\tau_{2 s-1}^{i}$ & Bus waiting time at station s; \\
\hline$\tau_{2 s}^{i}$ & Bus traveling time between station $\mathrm{s}$ and $\mathrm{s}+1 ;$ \\
\hline$\delta$ & Time needed for a passenger to get on/off the bus. \\
\hline
\end{tabular}

observed in the other stations of the model $\left(\mathrm{S}_{3}^{i}, \ldots, \mathrm{S}_{p_{i}}^{i}\right)$. The place $\mathrm{Pd}_{p_{i}, j}^{i}$ models the return path of the bus $\mathrm{j}$ from $\mathrm{S}_{p_{i}}^{i}$ to $\mathrm{S}_{1}^{i}$. A token in this place means that the bus $\mathrm{j}$ is going back to its departure station $\mathrm{S}_{1}^{i}$.

\section{2. $\quad$ Modeling passengers arrival and boarding using SPN}

Given that passengers arrival to their departure stations are usually random, so we have modeled them using a stochastic transition endowed with a stochastic distribution of rate denoted $\lambda$. In addition, we point out that the $\lambda$ parameter depends on passengers flow predicted and it differs from period to another (peak and off-peak periods for example).

The waiting passengers at a station $\mathrm{s}$ get on a bus $\mathrm{j}$ when this bus has empty seats inside. However, if it is full, passengers wait for the next one. As a matter of fact, we suppose that passengers boarding is made by FIFO (First In First Out) rule. It means that the first passenger who arrives to a station is the first one who gets on the 
bus. According to the station type, the SPN models representing passengers arrival are illustrated in figure 3.

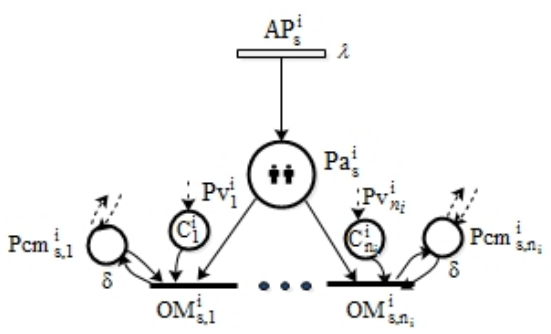

(a)

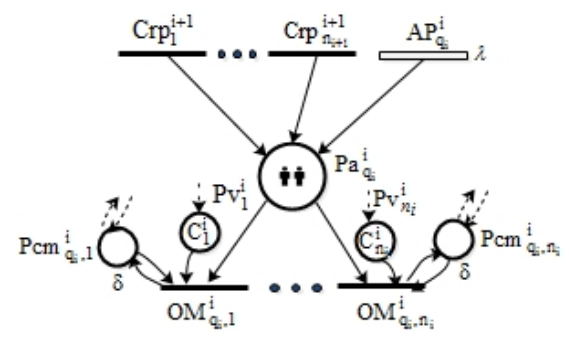

(b)

Figure 3. SPN model for passengers arrival and boarding

Table 2. Elements of SPN model for passengers arrival and boarding For $\mathrm{i}, \mathrm{j}$ and $\mathrm{s}\left(1 \leq \mathrm{i}, 1 \leq \mathrm{j} \leq \mathrm{n}_{i}, 1 \leq \mathrm{s} \leq \mathrm{p}_{i}\right)$

\begin{tabular}{l||l}
\hline \multicolumn{2}{l}{ Places } \\
\hline $\mathrm{Pcm}_{s, j}^{i}$ & Authorization for passengers to get on the bus $\mathrm{j} ;$ \\
\hline $\mathrm{Pa}_{s}^{i}$ & Passengers waiting at station s; \\
\hline $\mathrm{Pv}_{j}^{i}$ & Empty seats on the bus $\mathrm{j} ;$ \\
\hline $\operatorname{Transitions}^{i}$ \\
\hline $\mathrm{OM}_{s, j}^{i}$ & Passengers boarding to bus $\mathrm{j}$ at station $\mathrm{s} ;$ \\
\hline $\mathrm{AP}_{s}^{i}$ & Random passengers arrival at station s; \\
\hline $\mathrm{Crp}_{j^{\prime}}^{i+1}$ & Passengers correspondence (from $\mathrm{L}_{i+1}$ to $\mathrm{L}_{i}$ ) \\
\hline
\end{tabular}

- Case of terminus or intermediate stations $\left(\mathbf{S}_{s}^{i}, \mathbf{s}=\mathbf{1}, . ., \mathbf{p}_{i}, \mathbf{s} \neq \mathbf{q}_{i}\right)$

The figure 3-(a) describes passengers arrival to a terminus or intermediate stations. As explained before, the waiting passengers at a station $\mathrm{s}$ (tokens in $\mathrm{Pa}_{s}^{i}$ ) can get on the bus $\mathrm{j}$ (by firing the transition $\mathrm{OM}_{s, j}^{i}$ ) when they are authorized to get on (token in $\mathrm{Pcm}_{s, j}^{i}$ ) and there are empty seats inside (tokens in $\mathrm{Pv}_{j}^{i}$ ).

- Case of connection station (figure 3-(b))

In a connection station, passengers can come from other lines (in our case line $\mathrm{L}_{i+1}$ ). Passengers getting off a bus $\mathrm{j}^{\prime}$ of line $\mathrm{L}_{i+1}$ at the connection station have two choices: either make the correspondence with the line $\mathrm{L}_{i}$ or completely leave this station (the connection station is their destination). The transfer of passengers from line $\mathrm{L}_{i+1}$ to line $\mathrm{L}_{i}$ is modeled by the transitions $\operatorname{Crp}_{j^{\prime}}^{i+1}$ (where $\mathrm{j}^{\prime}$ is a bus of line $\mathrm{L}_{i+1}$ with $1 \leq j^{\prime} \leq \mathrm{n}_{i+1}$ ) (figure $3-(\mathrm{b})$ ).

\subsection{Modeling passengers destination choice and disembarking using $F C P N$}

Every passenger has his own destination (among $\mathrm{p}_{i}$ possible destinations). After arriving at their arrival station, passengers can disembark and leave the station or can make the correspondence with the other line. The PN model with conflicts that represents destination choice criteria is given in the following figure.

When the place $\mathrm{Pb}_{j}^{i}$ contains a token which models a passenger on the bus $\mathrm{j}$, there is a free choice in this place. So, the transition that will be fired can be chosen 


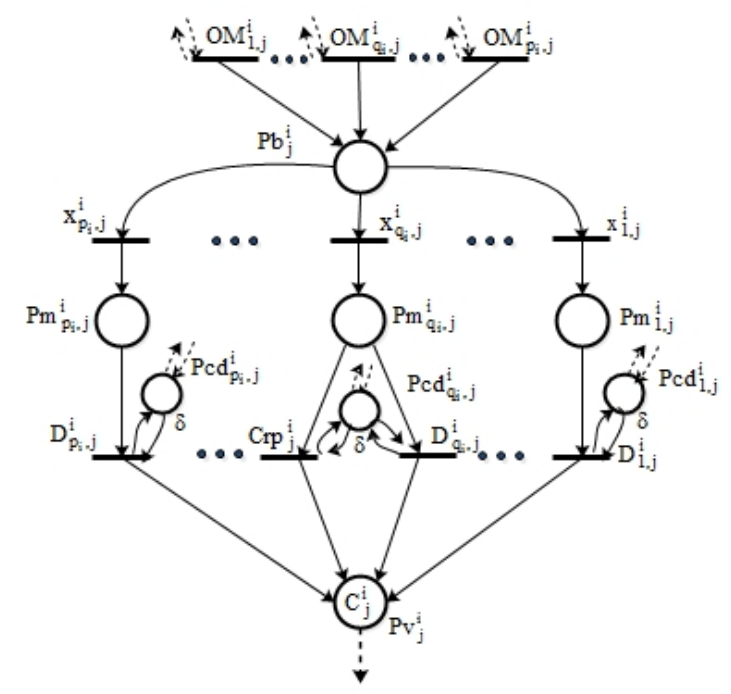

Figure 4. FCPN for modeling passengers destination choice and disembarking

Table 3. Elements of FCPN for modeling passengers destination choice and disembarking For $\mathrm{i}, \mathrm{j}$ and $\mathrm{s}\left(1 \leq \mathrm{i}, 1 \leq \mathrm{j} \leq \mathrm{n}_{i}, 1 \leq \mathrm{s} \leq \mathrm{p}_{i}\right)$

\begin{tabular}{l||l}
\hline \multicolumn{2}{l}{ Places } \\
\hline $\mathrm{Pcd}_{s, j}^{i}$ & Authorization for passengers to get off the bus $\mathrm{j} ;$ \\
\hline $\mathrm{Pm}_{s, j}^{i}$ & Passengers on board who will get off at station $\mathrm{s} ;$ \\
\hline $\mathrm{Pb}_{j}^{i}$ & Passengers boarding to bus $\mathrm{j} ;$ \\
\hline $\mathrm{Pv}_{j}^{i}$ & Empty seats on the bus $\mathrm{j} ;$ \\
\hline $\mathrm{Transit}^{i}$ ions \\
\hline $\mathrm{x}_{s, j}^{i}$ & Choice of station $\mathrm{s}$ as a destination; \\
\hline $\mathrm{Crp}_{j}^{i}$ & Passengers making correspondence with line $\mathrm{L}_{i+1} ;$ \\
\hline $\mathrm{D}_{s}^{i}$ & Passengers getting off the bus $\mathrm{j}$ at station $\mathrm{s} ;$ \\
\hline $\mathrm{OM}_{s, j}^{i}$ & Passengers boarding to bus $\mathrm{j}$ at station $\mathrm{s} ;$ \\
\hline
\end{tabular}

arbitrarily according to the passenger's destination. A transition $\mathrm{x}_{s, j}^{i}$ will be fired when a passenger has chosen station $\mathrm{s}\left(\mathrm{L}_{i}\right)$ as his destination. Furthermore, if a passenger wants to make a correspondence with the line $\mathrm{L}_{i+1}$, the transition $\mathrm{x}_{q_{i}, j}^{i}$ will be fired. The transition $\mathrm{D}_{s, j}^{i}$ models passengers disembarking from the bus $\mathrm{j}$ at station $\mathrm{s}$ without making correspondence. However, the transition $\mathrm{Crp}_{j}^{i}$ models passengers disembarking from the bus $\mathrm{j}$ at the connection station and making the correspondence with line $\mathrm{L}_{i+1}$. Finally, passengers release their seats (by adding tokens in the place $\mathrm{Pv}_{j}^{i}$ ) after getting off the bus (by firing $\mathrm{D}_{s, j}^{i}$ or $\operatorname{Crp}_{j}^{i}$ ).

The following section proposes the PN model of the line $\mathrm{L}_{i}$. It combines the three previous PN models that represent the main components of the system.

\subsection{Petri net model of the line $L_{i}$}

The global PN model of the line $\mathrm{L}_{i}$ combines all the PN models presented before (figure 2,3 and 4). We recall that the line $\mathrm{L}_{i}$ contains $\mathrm{p}_{i}$ stations and served by $\mathrm{n}_{i}$ buses. The global model presented in figure 5 consists of $n_{i}$ sub-models. Each sub-model represents a bus circuit with passengers destination choice. In this figure, we have 


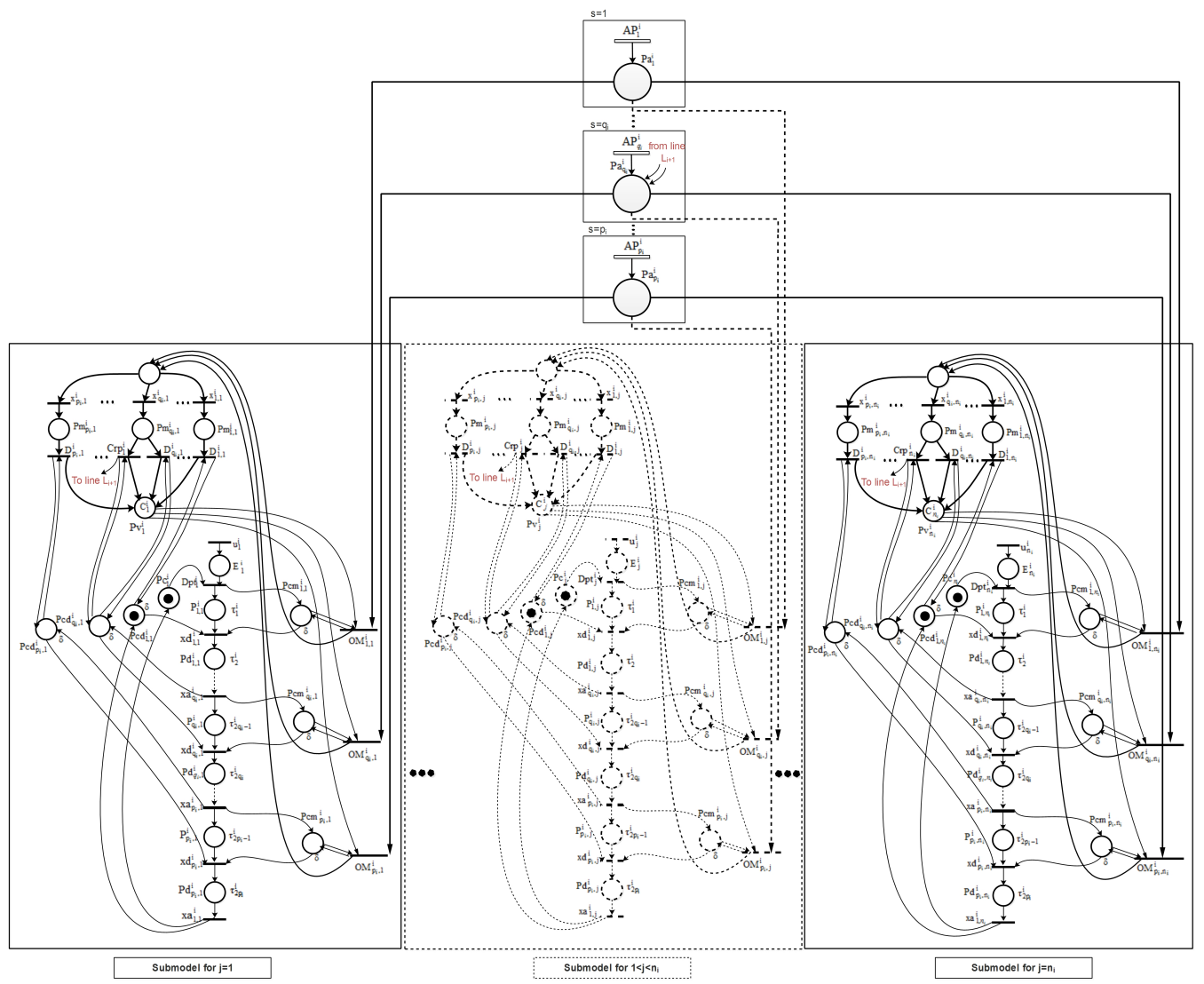

Figure 5. Petri net model of line $\mathrm{L}_{i}$ with $\mathrm{n}_{i}$ buses

represented three sub-models corresponding to $\mathrm{j}=1, \mathrm{j}=\mathrm{n}_{i}$ and the other buses (dotted part). The interaction of these sub-models is done by passengers waiting places $\mathrm{Pa}_{s}^{i}$ associated to their upstream transitions $\mathrm{AP}_{s}^{i}$ (and also $\operatorname{Crp}_{1}^{i+1}, \ldots$, and $\operatorname{Crp}_{n_{i+1}}^{i+1}$ in case of $\mathrm{s}=\mathrm{q}_{i}$ ). Furthermore, we obtain the same model for the line $\mathrm{L}_{i+1}$ while replacing the index $\mathrm{i}$ by the index $\mathrm{i}+1$. The transfer of passengers between the two lines $\mathrm{L}_{i}$ and $\mathrm{L}_{i+1}$ can take place at the connection station $\left(\mathrm{S}_{q_{i}}^{i}\right.$ or $\left.\mathrm{S}_{q_{i+1}}^{i+1}\right)$. The transitions that model passengers correspondence from $\mathrm{L}_{i+1}$ to $\mathrm{L}_{i}$ (resp. from $\mathrm{L}_{i}$ to $\mathrm{L}_{i+1}$ ) are $\mathrm{Crp}_{1}^{i+1}$,.., and $\operatorname{Crp}_{n_{i+1}}^{i+1}$ (resp. $\operatorname{Crp}_{1}^{i}, \ldots$, and $\operatorname{Crp}_{n_{i}}^{i}$ ). The advantage of these models, compared to other models established in the literature, is that it represents the state of all buses (moving, stopping, etc.) and all stations (empty, full, number of waiting passengers, etc.). In addition, all passengers arrival, boarding, destination choice, disembarking and correspondence are modeled.

In the next section, a $(\max ,+)$ model representing the behavior of the previous PN model is given. Moreover, some functions called routing functions are introduced to manage and solve all the encountered conflicts on the PN model.

\section{Modeling using $(\max ,+)$ algebra}

In this section, the $(\max ,+)$ models for both bus circuit and passengers boarding/disembarking are presented. We recall that $(\max ,+)$ dioid is the dioid $\mathbb{R}_{\max }=\mathbb{R} \cup$ $\{-\infty\}$ with two binary operations $\oplus$ and $\otimes$, where $\mathrm{a} \oplus \mathrm{b}$ is the maximum of a and $\mathrm{b}$, 
and $\mathrm{a} \otimes \mathrm{b}$ is the sum of $\mathrm{a}$ and $\mathrm{b}$, for all $\mathrm{a}, \mathrm{b} \in \mathbb{R}_{\max }$. The additive and multiplicative neutral elements are respectively $\varepsilon=-\infty$ and $e=0$. For more details about dioid algebra, especially $(\max ,+)$ algebra, the reader can refer to Baccelli et al. (1992).

It's well known that $(\max ,+)$ algebra is dedicated to the management and calculus of the occurrence dates of events (called "daters"). So, the associated dater with a transition $\mathrm{x}$ is denoted $\mathrm{x}(\mathrm{k})$ and represents the date of the $\mathrm{k}^{\text {th }}$ firing of $\mathrm{x}$.

\section{1. (Max, +) linear representations}

\subsection{1. (Max, +) model of bus circuit}

First of all, we describe the dynamic behavior of the $\mathrm{n}_{i}$ buses (figure 2 and 5 ), in terms of daters (arrival and departure times at different stops), by mathematical equations in the usual algebra and then by $(\max ,+)$ equations. So, the $\mathrm{k}^{\text {th }}$ firing of each transition of our PN model ( $\mathrm{k}^{\text {th }}$ turn or passage of the buses), described in figure 2 , is given by the following system.

for $j \in\left\{1, \ldots ., n_{i}\right\}$ and $\forall k>1$,

$$
\left\{\begin{array}{l}
D p t_{j}^{i}(k)=\max \left(u_{j}^{i}(k), x a_{1, j}^{i}(k-1)\right) \\
x d_{1, j}^{i}(k)=\max \left(D p t_{j}^{i}(k)+\tau_{1}^{i}, O M_{1, j}^{i}\left(k_{1, j}^{i}\right)+\delta, D_{1, j}^{i}\left(o_{1, j}^{i}\right)+\delta, x a_{1, j}^{i}(k-1)+\delta\right) \\
x a_{2, j}^{i}(k)=x d_{1, j}^{i}(k)+\tau_{2}^{i} \\
x d_{2, j}^{i}(k)=\max \left(x a_{2, j}^{i}(k)+\tau_{3}^{i}, O M_{2, j}^{i}\left(k_{2, j}^{i}\right)+\delta, D_{2, j}^{i}\left(o_{2, j}^{i}\right)+\delta\right) \\
\cdots \\
x a_{q_{i}, j}^{i}(k)=x d_{q_{i}-1, j}^{i}(k)+\tau_{2 q_{i}-2}^{i} \\
x d_{q_{i}, j}^{i}(k)=\max \left(x a_{q_{i}, j}^{i}(k)+\tau_{2 q_{i}-1}^{i}, O M_{q_{i}, j}^{i}\left(k_{q_{i}, j}^{i}\right)+\delta, D_{q_{i}, j}^{i}\left(o_{q_{i}, j}^{i}\right)+\delta, C r p_{j}^{i}\left(o_{p_{i}+1, j}^{i}\right)+\delta\right) \\
\cdots \\
x a_{p_{i}, j}^{i}(k)=x d_{p_{i}-1, j}^{i}(k)+\tau_{2\left(p_{i}-1\right)}^{i} \\
x d_{p_{i}, j}^{i}(k)=\max \left(x a_{p_{i}, j}^{i}(k)+\tau_{2 p_{i}-1}^{i}, O M_{p_{i}, j}^{i}\left(k_{p_{i}, j}^{i}\right)+\delta, D_{p_{i}, j}^{i}\left(o_{p_{i}, j}^{i}\right)+\delta\right) \\
x a_{1, j}^{i}(k)=x d_{p_{i}, j}^{i}(k)+\tau_{2 p_{i}}^{i}
\end{array}\right.
$$

- For the first equation of (1): every bus $\mathrm{j}$ starts its $\mathrm{k}^{t h}$ turn $\left(\mathrm{k}^{t h}\right.$ firing of $\mathrm{Dpt}_{j}^{i}$ ) after completing his $\mathrm{k}-1^{\text {th }}$ turn ( $\mathrm{k}-1^{\text {th }}$ firing of $\mathrm{xa}_{1, j}^{i}$ ), and a departure permission is given ( $\mathrm{k}^{\text {th }}$ firing of $\mathrm{u}_{j}^{i}$ ) (synchronization phenomenon).

- For buses departure daters (equations $\mathrm{xd}_{1, j}^{i}(\mathrm{k}), \ldots, \mathrm{xd}_{q_{i}, j}^{i}(\mathrm{k}), \ldots, \mathrm{xd}_{p_{i}, j}^{i}(\mathrm{k})$ of the system (1)): a bus $\mathrm{j}$ leaves a station $\mathrm{s}$ for the $\mathrm{k}^{\text {th }}$ time $\left(\mathrm{k}^{\text {th }}\right.$ firing of $\left.\mathrm{xd}_{s, j}^{i}\right)$ after waiting $\tau_{2 s-1}^{i}$ time units in this station so that passengers can get on (firing of $\mathrm{OM}_{s, j}^{i}$ ) and off (firing of $\mathrm{D}_{s, j}^{i}$ ) this bus. If several passengers are waiting, the FIFO rule is applied. The parameter $\mathrm{k}_{s, j}^{i}(\mathrm{k})\left(\right.$ resp. $\left.\mathrm{o}_{s, j}^{i}(\mathrm{k})\right)$ represents the number of firings of $\mathrm{OM}_{s, j}^{i}$ (resp. $\mathrm{D}_{s, j}^{i}$ ) before the $\mathrm{k}^{t h}$ departure of the bus $\mathrm{j}$ from the station $\mathrm{s}$ (see section 5.2.2 for more details). So, the expression $\mathrm{OM}_{s, j}^{i}\left(\mathrm{k}_{s, j}^{i}\right)$ (resp. $\left.\mathrm{D}_{s, j}^{i}\left(\mathrm{o}_{s, j}^{i}\right)\right)$ represents the last passenger boarding (resp. disembarking) time to/from the bus $\mathrm{j}$ ( $\mathrm{k}^{\text {th }}$ turn) at station $\mathrm{s}$.

- For buses arrival daters (equations $\mathrm{xa}_{1, j}^{i}(\mathrm{k}), . ., \mathrm{xa}_{q_{i}, j}^{i}(\mathrm{k}), . ., \mathrm{xa}_{p_{i}, j}^{i}(\mathrm{k})$ of the system $(1)$ ): a bus $\mathrm{j}$ arrives at a station $\mathrm{s}$ for the $\mathrm{k}^{\text {th }}$ time $\left(\mathrm{k}^{\text {th }}\right.$ firing of $\left.\mathrm{xa}_{s, j}^{i}\right)$ after $\tau_{2(s-1)}^{i}$ time units after leaving the station s-1.

Afterwards, we will try to simplify the previous system of equations in order to put it into a matrix form and solve it. Firstly, as we have mentioned in section 3, every bus $\mathrm{j}$ has to respect a scheduled timetable. So, the departure of each bus from every station must be independent from passengers arrival. Also, we assume that $\delta$ (time needed of get on/off a bus) is negligible compared to buses waiting times (for $1 \leq \mathrm{s} \leq \mathrm{p}_{i}$, 
$\left.\delta \ll \tau_{2 s-1}^{i}\right)$. Hence, the following relations are satisfied.

for $j \in\left\{1, \ldots, n_{i}\right\}, \forall k>1$,

$$
\left\{\begin{array}{l}
D p t_{j}^{i}(k)+\tau_{1}^{i}>O M_{1, j}^{i}\left(k_{1, j}^{i}\right)+\delta \\
x a_{2, j}^{i}(k)+\tau_{3}^{i}>O M_{2, j}^{i}\left(k_{2, j}^{i}\right)+\delta \\
\cdots \\
x a_{q_{i}, j}^{i}(k)+\tau_{2 q_{i}-1}^{i}>O M_{q_{i}, j}^{i}\left(k_{q_{i}, j}^{i}\right)+\delta \\
\cdots \\
x a_{p_{i}, j}^{i}(k)+\tau_{2 p_{i}-1}^{i}>O M_{p_{i}, j}^{i}\left(k_{p_{i}, j}^{i}\right)+\delta
\end{array}\right.
$$

Secondly, a bus j can leave a station s only after having disembarked all passengers who have this station s as destination. So, we have the following equations:

for $j \in\left\{1, \ldots ., n_{i}\right\}, \forall k>1$,

$$
\left\{\begin{array}{l}
D p t_{j}^{i}(k)+\tau_{1}^{i}>D_{1, j}^{i}\left(o_{1, j}^{i}\right)+\delta \\
x a_{2, j}^{i}(k)+\tau_{3}^{i}>D_{2, j}^{i}\left(o_{2, j}^{i}\right)+\delta \\
\cdots \\
x a_{q_{i}, j}^{i}(k)+\tau_{2 q_{i}-1}^{i}>D_{q_{i}, j}^{i}\left(o_{q_{i}, j}^{i}\right)+\delta \\
x a_{q_{i}, j}^{i}(k)+\tau_{2 q_{i}-1}^{i}>C r p_{j}^{i}\left(o_{p_{i}+1, j}^{i}\right)+\delta \\
\cdots \\
x a_{p_{i}, j}^{i}(k)+\tau_{2 p_{i}-1}^{i}>D_{p_{i}, j}^{i}\left(o_{p_{i}, j}^{i}\right)+\delta
\end{array}\right.
$$

Using (max, +) notations and considering the equations of (2) and (3), the system (1) can be simplified as follows:

for $j \in\left\{1, \ldots, n_{i}\right\}, \forall k>1$,

$$
\left\{\begin{array}{l}
D p t_{j}^{i}(k)=u_{j}^{i}(k) \oplus x a_{1, j}^{i}(k-1) \\
x d_{1, j}^{i}(k)=D p t_{j}^{i}(k) \otimes \tau_{1}^{i} \\
x a_{2, j}^{i}(k)=x d_{1, j}^{i}(k) \otimes \tau_{2}^{i} \\
x d_{2, j}^{i}(k)=x a_{2, j}^{i}(k) \otimes \tau_{3}^{i} \\
\cdots \\
x a_{q_{i}, j}^{i}(k)=x d_{q_{i}-1, j}^{i}(k) \otimes \tau_{2 q_{i}-2}^{i} \\
x d_{q_{i}, j}^{i}(k)=x a_{q_{i}, j}^{i}(k) \otimes \tau_{2 q_{i}-1}^{i} \\
\cdots \\
x a_{p_{i}, j}^{i}(k)=x d_{p_{i}-1, j}^{i}(k) \otimes \tau_{2\left(p_{i}-1\right)}^{i} \\
x d_{p_{i, j}}^{i}(k)=x a_{p_{i}, j}^{i}(k) \otimes \tau_{2 p_{i}-1}^{i}(k) \\
x a_{1, j}^{i}(k)=x d_{p_{i}, j}^{i}(k) \otimes \tau_{2 p_{i}}^{i}
\end{array}\right.
$$

In order to solve this system, we put it in a matrix form. To do so, we define the bus state vector $\mathrm{X}^{j}(\mathrm{k})$ and the input vector $\mathrm{U}^{j}(\mathrm{k})$ :

$\left\{\begin{array}{l}X^{j}(k)=\left[D p t_{j}^{i}(k), x d_{1, j}^{i}(k), x a_{2, j}^{i}(k), x d_{2, j}^{i}(k), x a_{3, j}^{i}(k), x d_{3, j}^{i}(k), . ., x a_{p_{i}, j}^{i}(k), x d_{p_{i}, j}^{i}(k), x a_{1, j}^{i}(k)\right]^{T} ; \\ U^{j}(k)=u_{j}^{i}(k) .\end{array}\right.$

Using these vectors, we can evaluate buses timetables for a given period.

The system (4) can then be written as the following matrix form:

$$
X^{j}(k)=A_{1} \otimes X^{j}(k) \oplus A_{2} \otimes X^{j}(k-1) \oplus B_{1} \otimes U^{j}(k)
$$

With : $\mathrm{A}_{1}, \mathrm{~A}_{2} \in \mathbb{R}_{\max }^{\left(2 p_{i}+1\right) \times\left(2 p_{i}+1\right)}$ and $\mathrm{B}_{1} \in \mathbb{R}_{\max }^{\left(2 p_{i}+1\right) \times(1)}$ 


$$
\mathrm{A}_{1}=\left[\begin{array}{ccccc}
\varepsilon & \varepsilon & \ldots & \varepsilon & \varepsilon \\
\tau_{1}^{i} & \varepsilon & \ldots & \varepsilon & \varepsilon \\
\varepsilon & \tau_{2}^{i} & \ldots & \varepsilon & \varepsilon \\
\vdots & \vdots & \ddots & \vdots & \vdots \\
\varepsilon & \varepsilon & \varepsilon & \tau_{2 p_{i}}^{i} & \varepsilon
\end{array}\right], \mathrm{A}_{2}=\left[\begin{array}{cccc}
\varepsilon & \ldots & \varepsilon & e \\
\varepsilon & \ldots & \varepsilon & \varepsilon \\
\varepsilon & \ldots & \varepsilon & \varepsilon \\
\vdots & \ddots & \vdots & \vdots \\
\varepsilon & \ldots & \varepsilon & \varepsilon
\end{array}\right] \text { and } \mathrm{B}_{1}=\left[\begin{array}{c}
e \\
\varepsilon \\
\varepsilon \\
\vdots \\
\varepsilon
\end{array}\right]
$$

The strictly triangular matrix $\mathrm{A}_{1}$ is nilpotent witch means that: $\exists \mathrm{r}, \forall \mathrm{u} \geq \mathrm{r} \mathrm{A}_{1}^{\otimes u}=\varepsilon$ (in this case $r=2 p_{i}+1$ ). Thereafter, the expression of the Kleene star of $A_{1}$ becomes $\mathrm{A}_{1}^{*}=\mathrm{Id} \oplus \mathrm{A}_{1} \oplus \mathrm{A}_{1}^{\otimes 2} \oplus \ldots \oplus \mathrm{A}_{1}^{\otimes r-1}$.

The implicit equation (5) can then be rewritten as a recurrence equation of first order as given in what follows:

$\forall k>1$,

$$
\begin{aligned}
X^{j}(k) & =A_{1} \otimes X^{j}(k) \oplus A_{2} \otimes X^{j}(k-1) \oplus B_{1} \otimes U^{j}(k) \\
& =A_{1} \otimes\left[A_{1} \otimes X^{j}(k) \oplus A_{2} \otimes X^{j}(k-1) \oplus B_{1} \otimes U^{j}(k)\right] \oplus A_{2} \otimes X^{j}(k-1) \oplus B_{1} \otimes U^{j}(k) \\
& =\ldots \ldots \ldots \ldots \ldots \ldots . . \\
& =A_{1}^{\otimes r} \otimes X^{j}(k) \oplus\left[A_{1}^{\otimes r-1} \oplus A_{1}^{\otimes r-2} \oplus \ldots \ldots . \oplus d\right] \otimes A_{2} \otimes X^{j}(k-1) \oplus A_{1}^{*} \otimes B_{1} \otimes U^{j}(k) \\
& =A_{1}^{*} \otimes A_{2} \otimes X^{j}(k-1) \oplus A_{1}^{*} \otimes B_{1} \otimes U^{j}(k)
\end{aligned}
$$

Therefore: $\forall k>1$,

$$
X^{j}(k)=A \otimes X^{j}(k-1) \oplus B \otimes U^{j}(k)
$$

With:

$$
\mathrm{A}=\mathrm{A}_{1}^{*} \otimes \mathrm{A}_{2}=\left[\begin{array}{cccc}
\varepsilon & \ldots & \varepsilon & e \\
\varepsilon & \ldots & \varepsilon & \theta_{1} \\
\varepsilon & \cdots & \varepsilon & \theta_{2} \\
\vdots & \ddots & \vdots & \vdots \\
\varepsilon & \ldots & \varepsilon & \theta_{2 p_{i}}
\end{array}\right], \mathrm{B}=\mathrm{A}_{1}^{*} \otimes \mathrm{B}_{1}=\left[\begin{array}{c}
e \\
\theta_{1} \\
\theta_{2} \\
\vdots \\
\theta_{2 p_{i}}
\end{array}\right]
$$

where:

$\theta_{l}=\bigotimes_{k=1}^{i} \tau_{k}^{i}$ for $\mathrm{l} \in\left\{1, . ., 2 \mathrm{p}_{i}\right\}$

\subsection{2. (Max, + ) model for passengers boarding}

In this section, $(\max ,+)$ equations for passengers boarding time are presented. The difficulty in expressing these equations appears in finding the different relations between the firing of the concerned transitions (see figure 3), namely $\mathrm{OM}_{s, j}^{i}$ and $\mathrm{AP}_{s}^{i}$ (and $\mathrm{Crp}_{1}^{i+1}, \ldots, \mathrm{Crp}_{n_{i+1}}^{i+1}$ in case of $\mathrm{s}=\mathrm{q}_{i}$ ). To overcome this main problem, we introduce some functions called routing functions (namely $\alpha_{s, j}^{i}(k), \beta_{s, j}^{i}(k)$ and $\delta_{u, j}^{i}(k)(\forall u \in$ $\left.\left.\left\{1, \ldots, \mathrm{n}_{i+1}+1\right\}\right)\right)$ to manage and solve the conflicts related to passengers boarding.

First of all, in case of terminus or intermediate stations $\left(\mathrm{s} \neq \mathrm{q}_{i}\right)$ (figure $\left.3-(\mathrm{a})\right)$, the $\mathrm{k}^{\text {th }}$ firing of the transition $\mathrm{OM}_{s, j}^{i}$ occurs after the $\alpha_{s, j}^{i^{t h}}(k)$ firing of the transition $\mathrm{AP}_{s}^{i}$ and $\beta_{s, j}^{i^{t h}}(k)$ firing of the transition $\mathrm{xa}_{s, j}^{i}$. Besides, in the connection station, passengers may come from the other line. For that reason, the $\mathrm{k}^{t h}$ firing of the transition $\mathrm{OM}_{s, j}^{i}$ occurs after $\beta_{s, j}^{i^{t h}}(k)$ firing of the transition $\mathrm{xa}_{s, j}^{i}$ and either the $\delta_{1, j}^{i^{t h}}(k)$ firing of $\mathrm{AP}_{s}^{i}$ or the $\delta_{2, j}^{i^{t h}}(k)$ (resp. or $\delta_{3, j}^{i^{t h}}(k), .$. , or $\delta_{n_{i+1}+1, j}^{i^{t h}}(k)$ ) firing of the transition $\operatorname{Crp}_{1}^{i+1}$ (resp. $\left.\mathrm{Crp}_{2}^{i+1}, \ldots, \operatorname{Crp}_{n_{i+1}}^{i+1}\right)$. The mathematical expression of these routing functions are given 
by the algorithm in figure 7 (equations (a), (b) and (c)).

The following formula allows to evaluate boarding time of each passenger at every station s.

For $\mathrm{s} \in\left\{1, . ., \mathrm{p}_{i}\right\}$ and $j \in\left\{1,2, \ldots, \mathrm{n}_{i}\right\}$

$\forall k \geq 1$,

$$
O M_{s, j}^{i}(k)= \begin{cases}A P_{s}^{i}\left(\alpha_{s, j}^{i}\right) \oplus O M_{s, j}^{i}(k-1) \cdot \delta \oplus x a_{s, j}^{i}\left(\beta_{s, j}^{i}\right) \cdot \delta \cdot 1_{s \neq 1}^{\max } \oplus D p t_{j}^{i}\left(\beta_{s, j}^{i}\right) \cdot \delta 1_{s=1}^{\max } & \text { if } s \neq q_{i}, \\ A P_{q_{i}}^{i}\left(\delta_{1, j}^{i}\right) \oplus{\stackrel{n_{i}+1}{\oplus}}_{m=2}^{\operatorname{man}} \operatorname{Cr} p_{m-1}^{i+1}\left(\delta_{m, j}^{i}\right) \oplus O M_{q_{i}, j}^{i}(k-1) \cdot \delta \oplus x a_{q_{i}, j}^{i}\left(\beta_{q_{i}, j}^{i}\right) \cdot \delta & \text { if } s=q_{i} .\end{cases}
$$

With:

- $\alpha_{s, j}^{i}(k)$ and $\delta_{u, j}^{i}(k)\left(\forall u \in\left\{1, \ldots, \mathrm{n}_{i+1}+1\right\}\right)$ represent the routing functions (given by the algorithm in figure 7, equations (a), (b) and (c)).

- $\beta_{s, j}^{i}(k)$ represents the turn of the bus $\mathrm{j}$ that will transport the $k^{\text {th }}$ passenger to his destination.

- The indicator function is given by the following equation.

$$
1_{c_{1}=c_{2}}^{\max }= \begin{cases}e & \text { if } c_{1}=c_{2} \\ \varepsilon & \text { if } c_{1} \neq c_{2}\end{cases}
$$

The routing functions mentioned earlier depend on some counters $\mathrm{k}_{s, j}^{i}(\mathrm{k})$ that represents the number of firings of $\mathrm{OM}_{s, j}^{i}$ before the $\mathrm{k}^{\text {th }}$ departure of the bus $\mathrm{j}$ from the station s. Let us first express these counters in what follows.

- Calculation of parameters $\mathbf{k}_{s, j}^{i}$ :

First, the buses are sent from their departure station one after the other (buses passage sequence is bus $\left.1,2, \ldots, \mathrm{n}_{i}, 1,2 \ldots\right)$. Second, given that buses have limited capacities then the counters $\mathrm{k}_{s, j}^{i}$ depends on $\mathrm{C}_{j}^{i}\left(\forall j \in\left\{1, \ldots, \mathrm{n}_{i}\right\}\right)$. For instance, if waiting passengers at station s exceeds the number of empty seats on the bus arriving to this station, then the number of passengers that are going to get on is the number of empty seats inside this bus (which justifies the operator "min" in equations below). The number of passengers arriving at station s from the beginning of buses journey (or number of firing of the transition $\mathrm{AP}_{s}^{i}$ with $\mathrm{s} \neq \mathrm{q}_{i}$ ) before the $\mathrm{k}^{\text {th }}$ departure of a bus $\mathrm{j}$ is given by $\mathrm{a}(\mathrm{k})=\sup _{p}\left\{A P_{s}^{i}(p)<x d_{s, j}^{i}(k)\right\}$. However, in a connection station, passengers can come from buses of line $\mathrm{L}_{i+1}$, so the number of these passengers at this station is given by $\mathrm{b}(\mathrm{k})=\sup _{p}\left\{A P_{q_{i}}^{i}(p)<x d_{q_{i}, j}^{i}(k)\right\}+\sum_{r=1}^{n_{i+1}} \sup _{p}\left\{C r p_{r}^{i+1}(p)<x d_{q_{i}, j}^{i}(k)\right\}$.

So, the equations of the counters $\mathrm{k}_{s, j}^{i}$ are given by: $\forall k \geq 1$

For $\mathrm{s} \neq \mathrm{q}_{i}$ (case of terminus or intermediate stations),

$$
k_{s, j}^{i}(k)= \begin{cases}\min \left[a(k), C_{j}^{i}+\sum_{m=1}^{s} k_{m, n_{i}}^{i}(k-1)-\sum_{m=1}^{s-1} k_{m, j}^{i}(k)+\sum_{m=1}^{s} D t_{m, j}^{i}(k)\right] & \text { if } j=1, \\ \min \left[a(k), C_{j}^{i}+\sum_{m=1}^{s} k_{m, j-1}^{i}(k)-\sum_{m=1}^{s-1} k_{m, j}^{i}(k)+\sum_{m=1}^{s} D t_{m, j}^{i}(k)\right] & \text { if } j \neq 1 .\end{cases}
$$


For $\mathrm{s}=\mathrm{q}_{i}$ (case of connection station),

$$
k_{q_{i}, j}^{i}(k)= \begin{cases}\min \left[b(k), C_{j}^{i}+\sum_{m=1}^{q_{i}} k_{m, n_{i}}^{i}(k-1)-\sum_{m=1}^{q_{i}-1} k_{m, j}^{i}(k)+\sum_{m=1}^{q_{i}} D t_{m, j}^{i}(k)\right] & \text { if } j=1, \\ \min \left[b(k), C_{j}^{i}+\sum_{m=1}^{q_{i}} k_{m, j-1}^{i}(k)-\sum_{m=1}^{q_{i}-1} k_{m, j}^{i}(k)+\sum_{m=1}^{q_{i}} D t_{m, j}^{i}(k)\right] & \text { if } j \neq 1 .\end{cases}
$$

With:

- $\mathrm{k}$ represents the $\mathrm{k}^{t h}$ turn of the bus $\mathrm{j}$. We recall that $\forall k \leq 0 \mathrm{k}_{s, j}^{i}(\mathrm{k})=0$

- $D t_{s, j}^{i}(k)$ represents the number of passengers who want to get off the bus $\mathrm{j}$ (in its $\mathrm{k}^{\text {th }}$ passage) at station $\mathrm{s}$. It includes the number of passengers who want to move from line $\mathrm{L}_{i}$ to line $\mathrm{L}_{i+1}$ (in case of $\mathrm{s}=\mathrm{q}_{i}$ ). This parameter is deduced from passengers destination choice.

The number of waiting passengers who will get on a bus $\mathrm{j} \neq 1$ (resp. $\mathrm{j}=1$ ) (in its $\mathrm{k}^{\text {th }}$ passage) at a station $\mathrm{s}$ is given by $\mathrm{k}_{s, j}^{i}(\mathrm{k})-\mathrm{k}_{s, j-1}^{i}(\mathrm{k})\left(\right.$ resp. $\left.\mathrm{k}_{s, 1}^{i}(\mathrm{k})-\mathrm{k}_{s, n_{i}}^{i}(\mathrm{k}-1)\right)$ and denoted $\mathrm{N}_{s, j}^{i}(\mathrm{k})$.

The algorithm, given by figure 6 , allows to manage occurred conflicts in figure 3 by computing the routing functions mentioned above. It is triggered when a bus $\mathrm{j}$ arrives to a station s. First of all, we compute the number of passenger who will get on the bus $\mathrm{j}$ (in its $\mathrm{r}^{\text {th }}$ passage) given by $\mathrm{N}_{s, j}^{i}(\mathrm{r})$. Afterwards, according to the type of station $\mathrm{s}$ (correspondence or intermediate or terminus), we compute the considered routing functions. The figure 7 , represent the same algorithm with more details. The expression of these routing functions are explicitly developed with mathematical equations.

In the next section, the $(\max ,+)$ equations describing passengers disembarking times are evaluated.

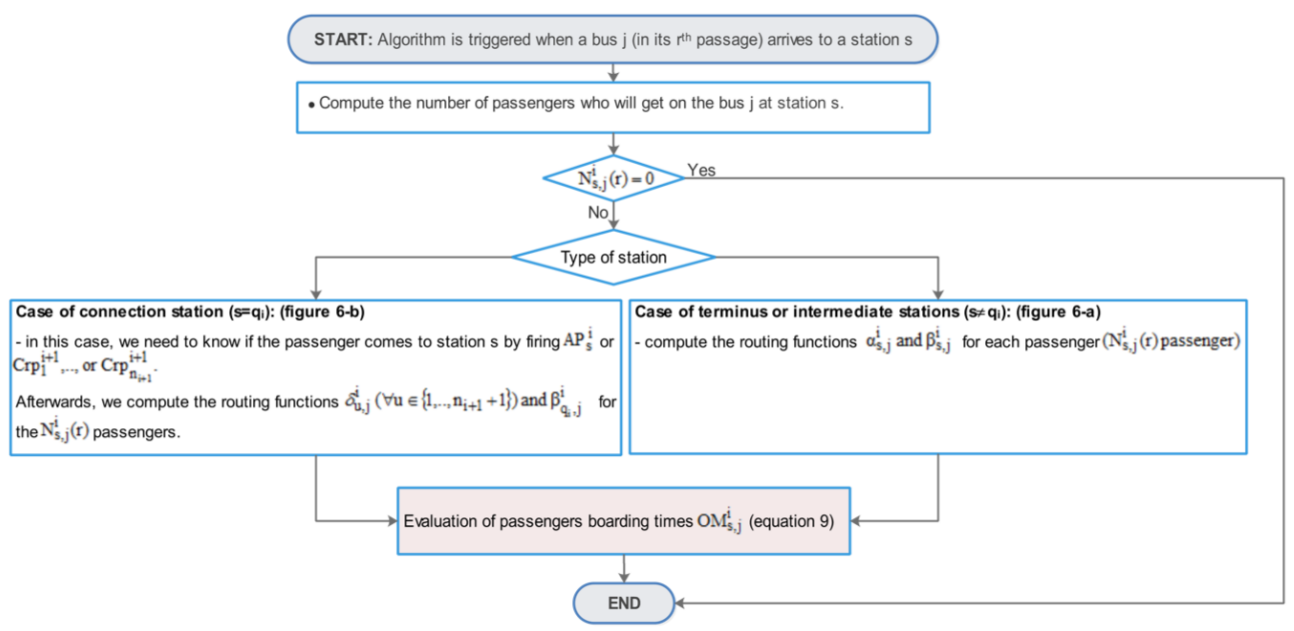

Figure 6. Flowchart for computing the routing functions related to passengers boarding

\subsection{3. (Max, +) model for passengers disembarking}

Passengers get off the bus $\mathrm{j}$ at station s (by firing the transitions $\mathrm{D}_{s, j}^{i}$ or $\operatorname{Crp}_{j}^{i}$ ) when these passengers have chosen station $\mathrm{s}$ as their destination (firing of transitions $\mathrm{x}_{s, j}^{i}$ ). So the $(\max ,+)$ equations describing passengers disembarking times from the bus $\mathrm{j}$ are given by: 


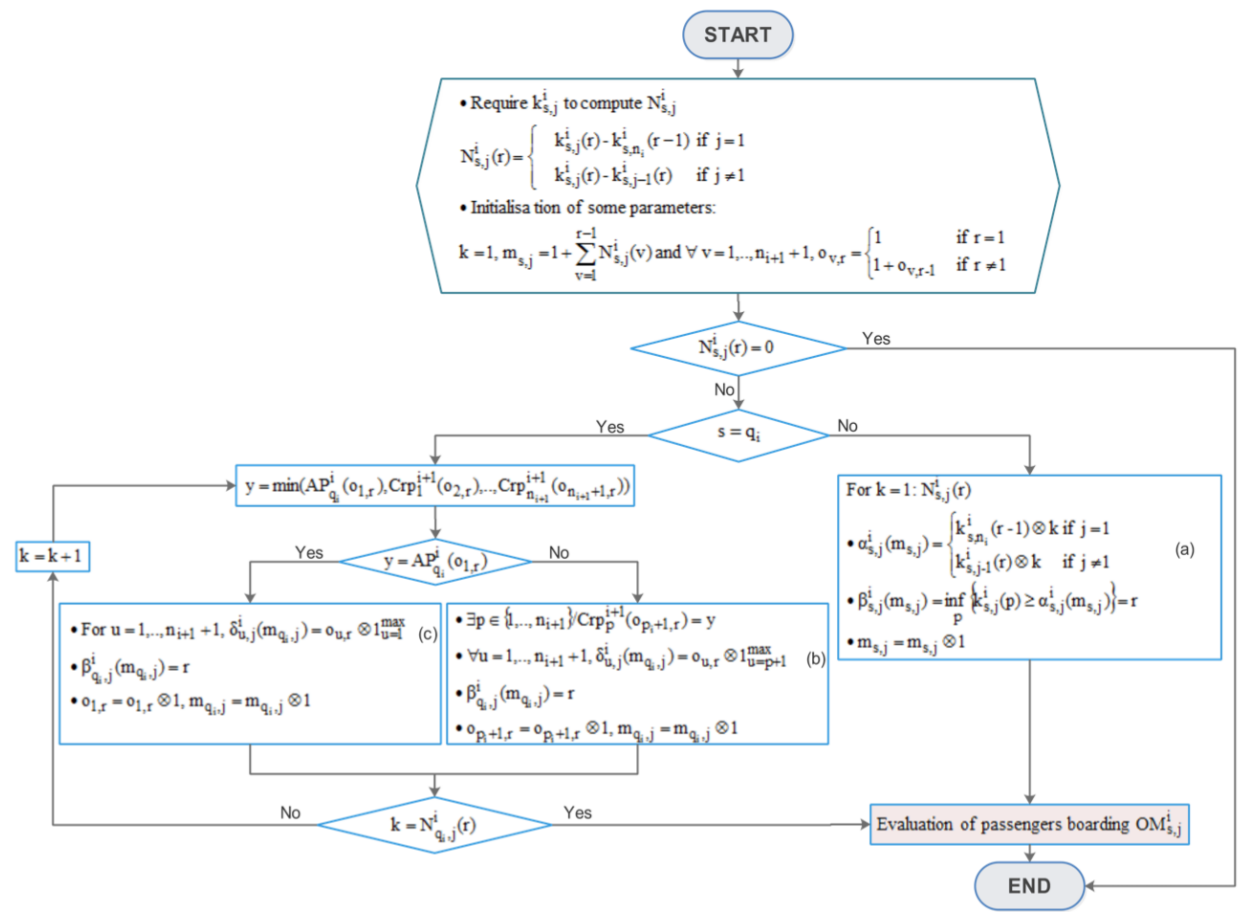

Figure 7. Detailed version of flowchart of Fig. 10 with explicit expressions of routing functions

$\forall k \geq 1$

$$
\left\{\begin{array}{l}
D_{1, j}^{i}(k)=x_{1, j}^{i}(k) \oplus x a_{1, j}^{i}\left(\xi_{p_{i}+1, j}^{i}\right) \otimes \delta \oplus D_{1, j}^{i}(k-1) \otimes \delta \\
D_{2, j}^{i}(k)=x_{2, j}^{i}(k) \oplus x a_{2, j}^{i}\left(\xi_{p_{i}^{2}+2, j}^{i}\right) \otimes \delta \oplus D_{2, j}^{i}(k-1) \otimes \delta \\
\ldots \\
D_{q_{i}, j}^{i}(k)=x_{q_{i}, j}^{i}\left(\xi_{p_{i}\left(p_{i}+1\right)+2, j}^{i}\right) \oplus x a_{q_{i}, j}^{i}\left(\xi_{p_{i}^{2}+q_{i}, j}^{i}\right) \otimes \delta \oplus D_{q_{i}, j}^{i}(k-1) \otimes \delta \oplus C r p_{j}^{i}\left(\xi_{p_{i}\left(p_{i}+1\right)+4, j}^{i}\right) \otimes \delta \\
\ldots \ldots \\
D_{p_{i}, j}^{i}(k)=x_{p_{i}, j}^{i}(k) \oplus x a_{p_{i}, j}^{i}\left(\xi_{p_{i}\left(p_{i}+1\right), j}^{i}\right) \otimes \delta \oplus D_{p_{i}, j}^{i}(k-1) \otimes \delta \\
C r p_{j}^{i}(k)=x_{q_{i}, j}^{i}\left(\xi_{p_{i}\left(p_{i}+1\right)+3, j}^{i}\right) \oplus x a_{q_{i}, j}^{i}\left(\xi_{p_{i}\left(p_{i}+1\right)+1, j}^{i}\right) \otimes \delta \oplus C r p_{j}^{i}(k-1) \otimes \delta \oplus D_{q_{i}, j}^{i}\left(\xi_{p_{i}\left(p_{i}+1\right)+5, j}^{i}\right) \otimes \delta
\end{array}\right.
$$

The routing functions $\xi_{u, j}^{i}\left(\mathrm{u} \in\left\{1, \ldots, p_{i}\left(p_{i}+1\right)+5\right\}\right.$ are proposed to solve all the conflicts and concurrency in figure 4 . These functions depends essentially on passengers destination.

In the first equation of (11), the $\mathrm{k}^{\text {th }}$ passenger, who has chosen departure station as his destination (by firing of $\mathrm{x}_{1, j}^{i}$ ), get off the bus $\mathrm{j}$ at the departure station ( $\mathrm{k}^{\text {th }}$ firing of the transition $\mathrm{D}_{1, j}^{i}$ ) when the bus $\mathrm{j}$, in its $\xi_{p_{i}^{2}+1, j}^{i^{t h}}$ passage, arrives to this station. Furthermore, if several passengers want to get off, the FIFO rule is applied. The other equations are expressed in the same way.

The firing dates of $\mathrm{x}_{s, j}^{i}$ depends on passengers destination and boarding times and they are given by the following equations: $\forall k \geq 1$,

$$
\left\{\begin{array}{l}
x_{1, j}^{i}(k)=O M_{1, j}^{i}\left(\xi_{1, j}^{i}\right) \oplus O M_{2, j}^{i}\left(\xi_{2, j}^{i}\right) \oplus O M_{3, j}^{i}\left(\xi_{3, j}^{i}\right) \oplus \ldots \oplus O M_{p_{i}, j}^{i}\left(\xi_{p_{i, j}}^{i}\right) \\
x_{2, j}^{i}(k)=O M_{1, j}^{i}\left(\xi_{p_{i}+1, j}^{i}\right) \oplus O M_{2, j}^{i}\left(\xi_{p_{i}+2, j}^{i}\right) \oplus O M_{3, j}^{i}\left(\xi_{p_{i}+3, j}^{i}\right) \oplus \ldots \oplus M_{p_{i}, j}^{i}\left(\xi_{2 p_{i}, j}^{i}\right) \\
\ldots . \\
x_{q_{i}, j}^{i}(k)=O M_{1, j}^{i}\left(\xi_{p_{i}\left(q_{i}-1\right)+1, j}^{i}\right) \oplus O M_{2, j}^{i}\left(\xi_{p_{i}\left(q_{i}-1\right)+2, j}^{i}\right) \oplus O M_{3, j}^{i}\left(\xi_{p_{i}\left(q_{i}-1\right)+3, j}^{i}\right) \oplus \ldots \oplus O M_{p_{i}, j}^{i}\left(\xi_{p_{i} q_{i}, j}^{i}\right) \\
\ldots . i \\
x_{p_{i}, j}^{i}(k)=O M_{1, j}^{i}\left(\xi_{p_{i}\left(p_{i}-1\right)+1, j}^{i}\right) \oplus O M_{2, j}^{i}\left(\xi_{p_{i}\left(p_{i}-1\right)+2, j}^{i}\right) \oplus O M_{3, j}^{i}\left(\xi_{p_{i}\left(p_{i}-1\right)+3, j}^{i}\right) \oplus \ldots \oplus O M_{p_{i}, j}^{i}\left(\xi_{p_{i}, j}^{i}\right)
\end{array}\right.
$$


So : for $\mathrm{s} \in\left\{1, . ., \mathrm{p}_{i}\right\}$ and $\mathrm{j} \in\left\{1, . ., \mathrm{n}_{i}\right\}, \forall k \geq 1$,

$$
x_{s, j}^{i}(k)=O M_{1, j}^{i}\left(\xi_{p_{i}(s-1)+1, j}^{i}\right) \oplus O M_{2, j}^{i}\left(\xi_{p_{i}(s-1)+2, j}^{i}\right) \oplus \ldots \oplus O M_{p_{i}, j}^{i}\left(\xi_{s . p_{i}, j}^{i}\right)=\bigoplus_{d=1}^{p_{i}} O M_{d, j}^{i}\left(\xi_{p_{i}(s-1)+d, j}^{i}\right)
$$

The system (11) can then be expressed as follows:

$$
D_{s, j}^{i}(k)= \begin{cases}x_{q_{i}, j}^{i}\left(\xi_{p_{i}\left(p_{i}+1\right)+2, j}^{i}\right) \oplus x a_{q_{i}, j}^{i}\left(\xi_{p_{i}^{2}+q_{i}, j}^{i}\right) \cdot \delta \oplus D_{q_{i}, j}^{i}(k-1) \cdot \delta \oplus C r p_{j}^{i}\left(\xi_{p_{i}\left(p_{i}+1\right)+4, j}^{i}\right) \cdot \delta & \text { if } s=q_{i}, \\ x_{s, j}^{i}(k) \oplus x a_{s, j}^{i}\left(\xi_{p_{i}^{2}+s, j}^{i}\right) \cdot \delta \oplus D_{s, j}^{i}(k-1) \cdot \delta & \text { if } s \neq q_{i} .\end{cases}
$$

With passengers correspondence dates are given by the following equation:

$$
\operatorname{Crp}_{j}^{i}(k)=x_{q_{i}, j}^{i}\left(\xi_{p_{i}\left(p_{i}+1\right)+3, j}^{i}\right) \oplus x a_{q_{i}, j}^{i}\left(\xi_{p_{i}\left(p_{i}+1\right)+1, j}^{i}\right) \cdot \delta \oplus \operatorname{Crp} p_{j}^{i}(k-1) \cdot \delta \oplus D_{q_{i}, j}^{i}\left(\xi_{p_{i}\left(p_{i}+1\right)+5, j}^{i}\right) \cdot \delta
$$

To evaluate disembarking of passengers at different stations, we have to solve the system of equations (14) and (15). To do so, the algorithm, presented in figure 8, is suggested to manage the conflicts and resources sharing in figure 4. In this algorithm, the random destination of $\mathrm{k}^{\text {th }}$ passenger, who get on a bus $\mathrm{j}$ at station $\mathrm{s}$, is given by $\operatorname{dest}_{s, j}^{i}(\mathrm{k})$. From this destination we can compute all the routing functions appearing in the equations (14) and (15). The algorithm in figure 9 is presented in order to give more details about the mathematical expression of these routing functions.

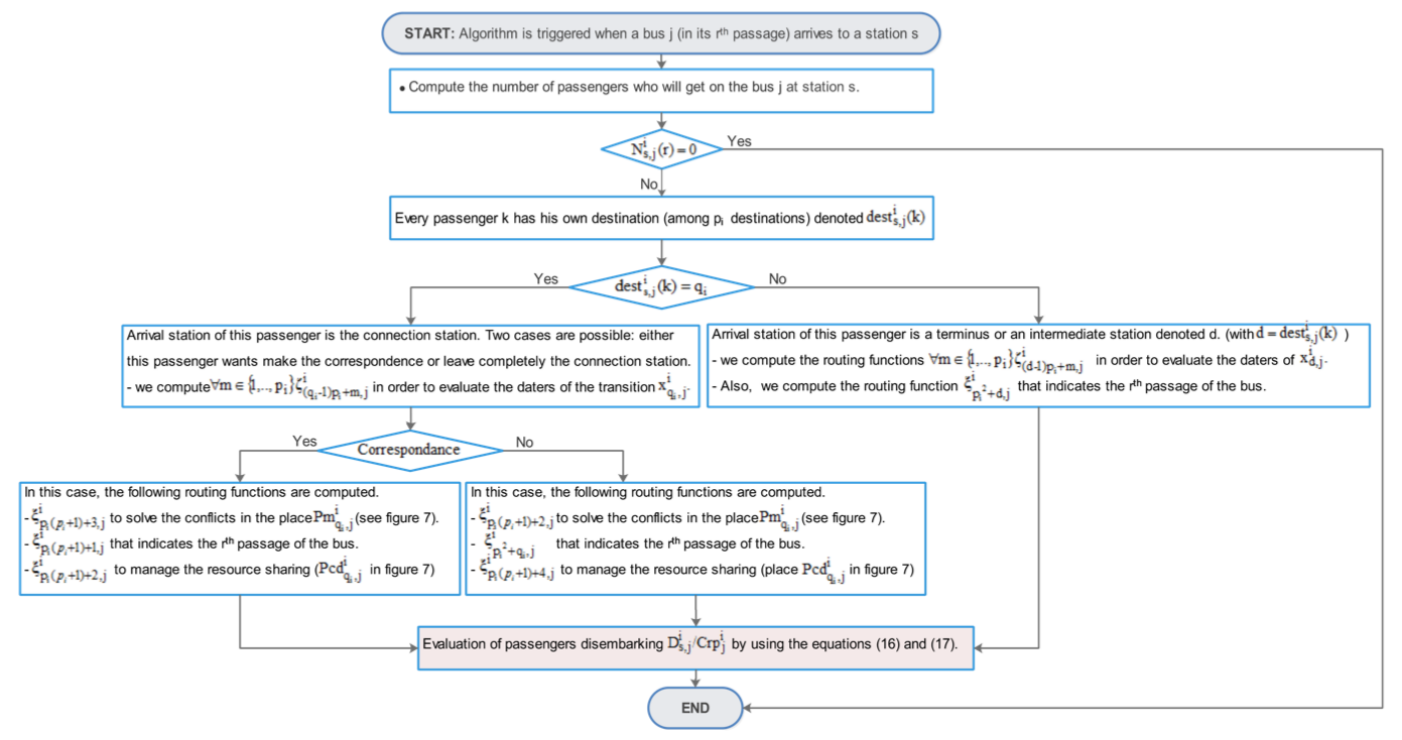

Figure 8. Flowchart for computing the routing functions related to passengers disembarking

In brief, the methodology adopted to evaluate the performances of the studied network is presented in figure 10. It consists of three related models: (i) buses circuit, (ii) passengers arrival and boarding and finally (iii) passengers destination choice and disembarking. In the first part, we developed a $(\max ,+)$ equation to evaluate buses timetables given by the vectors $\mathrm{X}_{j}^{i}\left(\forall j \in\left\{1, \ldots, \mathrm{n}_{i}\right\}\right)$. In the second part, given that passengers arrival are random, we introduce some routing functions to manage and solve the conflicts in figure 3 , in order to express passengers boarding by $(\max ,+)$ equations. Finally, the last part is dedicated to manage passengers destination choice in order to evaluate their disembarking times. 


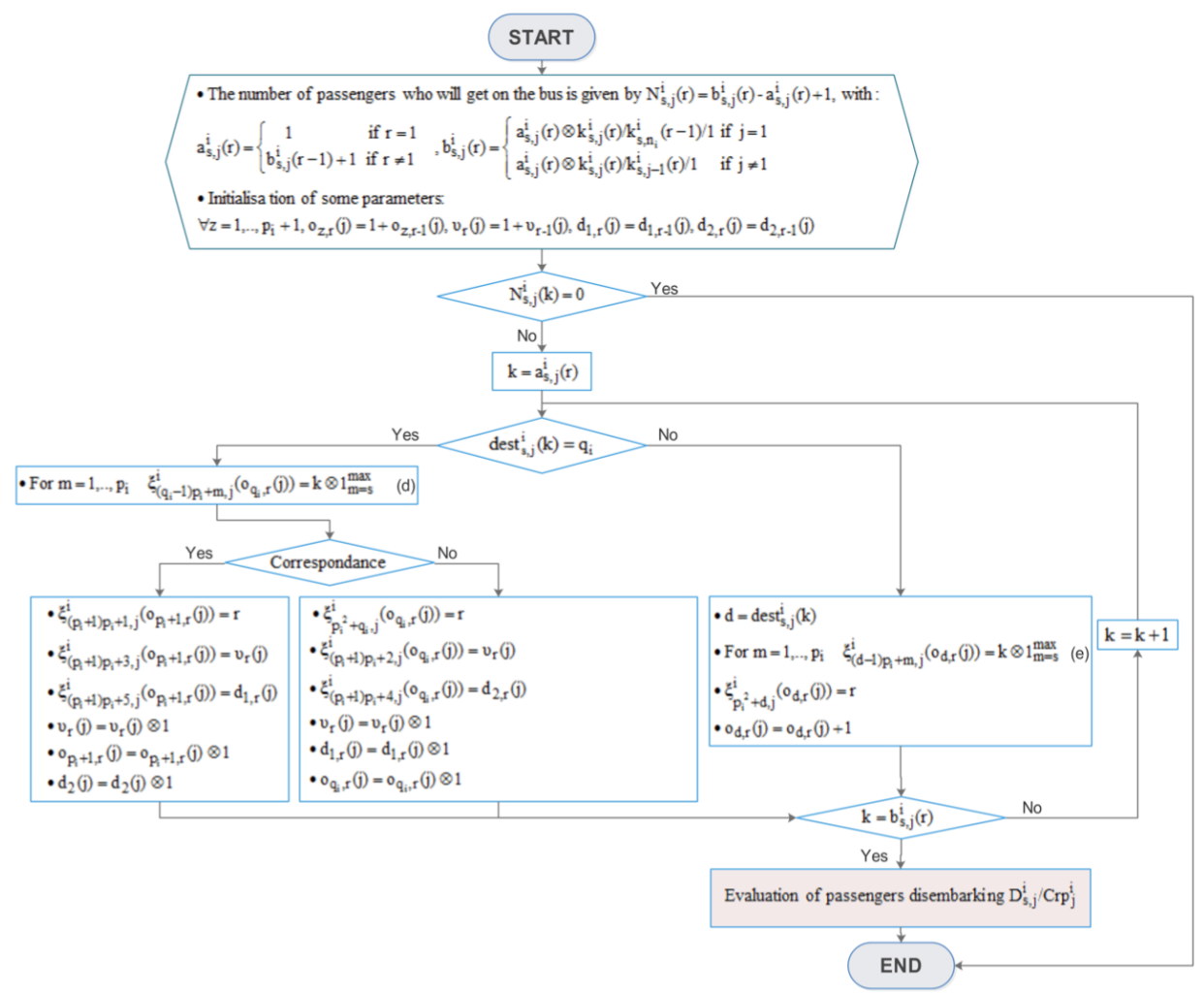

Figure 9. Detailed version of flowchart of Fig. 12 with explicit expressions of routing functions

\section{Performance evaluation and system control}

In this section, we are going to evaluate the performances of the bus network described in figure 1. More precisely, traveling time of each passenger is evaluated. Furthermore, the influence of the limited capacity of buses on passengers travel time is studied, in order to find an optimal capacity from which this capacity has no longer impact on passengers waiting time.

\subsection{Passengers traveling time evaluation}

As described before, for each passenger of the bus network presented in figure 1, we are able to determine the bus he will take and also his boarding and disembarking time (see equations (7), (14) and (15)). Moreover, the waiting time of the $\mathrm{k}^{\text {th }}$ arriving passenger who will take a bus $\mathrm{j}$ at his departure station $\mathrm{s}$ is given by: $\forall \mathrm{k} \geq 1$,

$$
T_{s, j}^{i}(k)=x d_{s, j}^{i}\left(\beta_{s, j}^{i}\right) / A P_{s}^{i}(k)
$$

where: $\mathrm{Ap}_{s}^{i}(\mathrm{k})$ is the arrival time of $\mathrm{k}^{\text {th }}$ passenger and $\mathrm{xd}_{s, j}^{i}\left(\beta_{s, j}^{i}\right)$ represents the departure time of the bus $\mathrm{j}$ that will transport this passenger. We recall that the operator " / expressed in $(\max ,+)$ algebra is equivalent to the subtraction in the usual algebra. However, if a passenger wants the make the correspondence with line $\mathrm{L}_{i+1}$, the waiting time of this passenger at the connection station is given by: $\forall \mathrm{k} \geq 1$,

$$
T_{j, j^{\prime}}(k)=x d_{q_{i+1}, j^{\prime}}^{i+1}\left(\beta_{q_{i+1}, j^{\prime}}^{i+1}\right) / x a_{q_{i}, j}^{i}\left(\beta_{q_{i}, j}^{i}\right)
$$




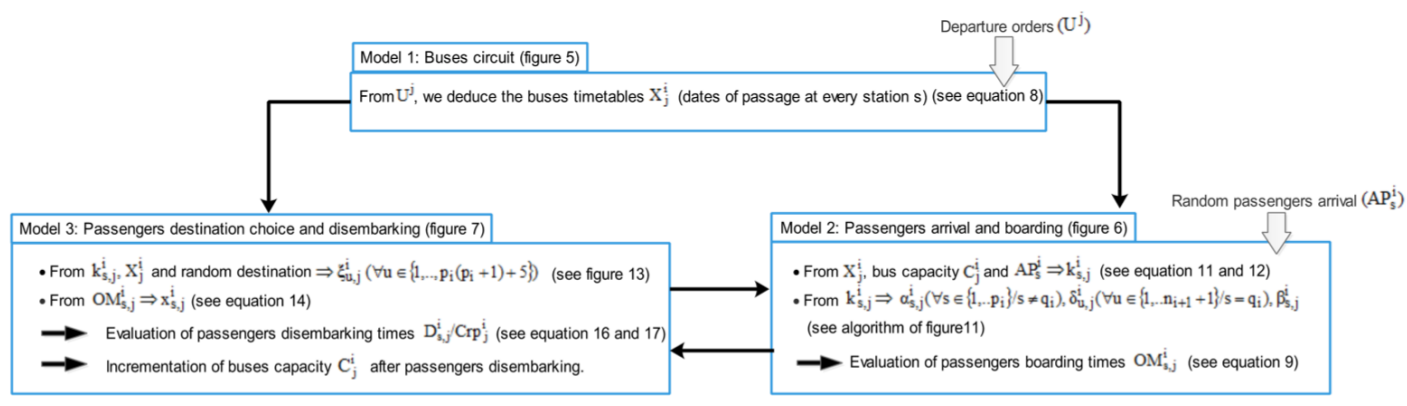

Figure 10. Interaction between the established models

where: $\mathrm{xa}_{q_{i}, j}^{i}\left(\beta_{q_{i}, j}^{i}\right)$ is the arrival time of the bus $\mathrm{j}$ (bus of line $\mathrm{L}_{i}$ ) to the connection station to let this passenger making the correspondence and $\operatorname{xd}_{q_{i+1}, j^{\prime}}^{i+1}\left(\beta_{q_{i+1}, j^{\prime}}^{i+1}\right)$ represents the departure time of the bus $\mathrm{j}^{\prime}$ (bus of line $\mathrm{L}_{i+1}$ ) that will transport this passenger from the connection station.

Finally, passengers traveling time is deduced from their arrival time $\left(\mathrm{AP}_{s}^{i}\right)$ and their disembarking time $\mathrm{D}_{s, j}^{i}$ (or $\mathrm{D}_{s^{\prime}, j^{\prime}}^{i+1}$ if a passenger wants to make a correspondence).

\subsection{Calculation of buses optimal capacity}

The optimal capacity refers to the maximum number of places considered in a bus to reach a point from which the capacity has no longer impact on passengers waiting time. As a result, if several types of bus with different capacities are available, this parameter indicates the one that will ensure the transportation service. The ultimate objective is choosing a bus corresponding to the number of passengers to be transported especially in peak periods. This will impact positively the transportation company costs and passengers waiting times.

Before determining the optimal capacity, we first simplify the expression of the counters $\mathrm{k}_{s, j}^{i}$. These parameters are calculated such a way that they no longer depend on buses capacities. It means that every passenger who come to a station s can get on the arriving bus (like if the capacity is infinite) regardless of the capacity. As described before, from these counters, we can deduce the number of waiting passengers at every station s including the connection one.

For $\mathrm{j} \in\left\{1, \ldots ., n_{i}\right\}$ and $\mathrm{s} \in\left\{1, . ., p_{i}\right\}$,

$\forall k \geq 1$

$$
k_{s, j}^{i}(k)= \begin{cases}\sup _{p}\left\{A P_{s}^{i}(p)<x d_{s, j}^{i}(k)\right\} & \text { if } s \neq q_{i}, \\ \sup _{p}\left\{A P_{q_{i}}^{i}(p)<x d_{q_{i}, j}^{i}(k)\right\}+\sum_{r=1}^{n_{i+1}} \sup _{p}\left\{C r p_{r}^{i+1}(p)<x d_{q_{i}, j}^{i}(k)\right\} & \text { if } s=q_{i} .\end{cases}
$$

The number of passengers on board of the bus $\mathrm{j}$ at the station $\mathrm{s}$ is expressed as follows $\forall k \geq 1$

$$
y_{s, j}^{i}(k)= \begin{cases}y_{s-1, j}^{i}(k)+k_{s, j}^{i}(k)-k_{s, j-1}^{i}(k)-D t_{s, j}^{i}(k) & \text { if } j \neq 1, \\ y_{s-1,1}^{i}(k)+k_{s, 1}^{i}(k)-k_{s, n_{i}}^{i}(k-1)-D t_{s, 1}^{i}(k) & \text { if } j=1 .\end{cases}
$$


Where: $\mathrm{k}$ represents the $\mathrm{k}^{\text {th }}$ turn of the bus, $\mathrm{Dt}_{s, j}^{i}(\mathrm{k})$ represents the number of passengers who want to get off the bus $\mathrm{j}$ at the station $\mathrm{s}$ and $\mathrm{k}_{s, j}^{i}(\mathrm{k})$ represents the counters defined above. The number of passengers on board depends on arrival dates of passengers (expressed by $\mathrm{AP}_{s}^{i}$ ). So, the optimal capacity of the bus $\mathrm{j}$ is given by the following equation :

$$
\operatorname{Copt}_{j}^{i}(k)=\bigoplus_{s=1}^{p_{i}} y_{s, j}^{i}(k)
$$

Given that passengers arrival suffers from a strong variation in real-time (peak and off-peak periods), the above-mentioned parameter is developed for each turn of the bus. The aim of calculating the optimum capacity is to determine the bus (from the fleet) that will transport all passengers waiting at every line station. This will enables all passengers to get on the bus without being constrained to wait the next one.

In order to illustrate the proposed approach, a numerical example is presented in the following section and the obtained results are reported and analyzed.

\section{Numerical example}

In this section, we consider the following numerical example (table 4) of the bus network of figure 1. It is important to note that the studied case is a sub-system of a global bus network. This sub-system is a zoom of a real-world example. Without losing the generality, the proposed approach can be applied on more complex transportation systems (bus, train, subway, ....) with more connected lines and correspondence stations. Our current research work in this direction, is about the generalization of this approach. Preliminary obtained results are satisfying and promising. The table below includes the data of the studied network.

Table 4. Data of the considered network

\begin{tabular}{c|c|c}
\hline Data & Line $\mathrm{L}_{i}$ & Line $\mathrm{L}_{i+1}$ \\
\hline Number of stations & $\mathrm{p}_{i}=3$ & $\mathrm{p}_{i+1}=4$ \\
\hline Bus waiting time $(\min )$ & $\tau_{1}^{i}=3, \tau_{3}^{i}=2, \tau_{5}^{i}=1$ & $\tau_{1}^{i+1}=4, \tau_{3}^{i+1}=3, \tau_{5}^{i+1}=1, \tau_{7}^{i+1}=1$ \\
\hline Bus traveling time $(\min )$ & $\tau_{2}^{i}=23, \tau_{4}^{i}=25, \tau_{6}^{i}=45$ & $\tau_{2}^{i+1}=15, \tau_{4}^{i+1}=10, \tau_{6}^{i+1}=10, \tau_{8}^{i+1}=40$ \\
\hline Number of buses & $\mathrm{n}_{i}=2$ & $\mathrm{n}_{i+1}=3$ \\
\hline Buses capacity & $\mathrm{C}_{1}^{i}=40, \mathrm{C}_{2}^{i}=40$ & $\mathrm{C}_{1}^{i+1}=35, \mathrm{C}_{2}^{i+1}=35, \mathrm{C}_{3}^{i+1}=50$ \\
\hline Buses starting time $(\min )$ & $\mathrm{u}_{1}^{i}(1)=0, \mathrm{u}_{2}^{i}(1)=50$ & $\mathrm{u}_{1}^{i+1}(1)=0, \mathrm{u}_{2}^{i+1}(1)=35, \mathrm{u}_{3}^{i+1}(1)=70$ \\
\hline Other parameters & $\delta=0.1 \mathrm{~min}, \mathrm{q}_{i}=2$ and $\mathrm{q}_{i+1}=2$ \\
\hline
\end{tabular}

To simplify this study, we assume that: $\forall k \geq 1, \mathrm{u}_{j}^{i}(\mathrm{k})=\mathrm{xa}_{1, j}^{i}(\mathrm{k}-1)$ which means that as soon as a bus arrives to its departure station (achievement of a circuit), it will begin a new one. As a result, the equation (6) becomes:

$\forall k \geq 1$ and $\mathrm{j} \in\left\{1, . ., n_{i}\right\}$

$$
X^{j}(k)=A \otimes X^{j}(k-1)=A^{\otimes(k-1)} \otimes X^{j}(1)
$$

The characteristic matrix $\mathrm{A}$ of line $\mathrm{L}_{i}$ is given by: 


$$
A=\left[\begin{array}{lllllll}
\varepsilon & \varepsilon & \varepsilon & \varepsilon & \varepsilon & \varepsilon & e \\
\varepsilon & \varepsilon & \varepsilon & \varepsilon & \varepsilon & \varepsilon & 3 \\
\varepsilon & \varepsilon & \varepsilon & \varepsilon & \varepsilon & \varepsilon & 26 \\
\varepsilon & \varepsilon & \varepsilon & \varepsilon & \varepsilon & \varepsilon & 28 \\
\varepsilon & \varepsilon & \varepsilon & \varepsilon & \varepsilon & \varepsilon & 53 \\
\varepsilon & \varepsilon & \varepsilon & \varepsilon & \varepsilon & \varepsilon & 54 \\
\varepsilon & \varepsilon & \varepsilon & \varepsilon & \varepsilon & \varepsilon & 99
\end{array}\right]
$$

After implementing the proposed algorithms while using the numerical data of table 4 , we obtain the results reported in what follows.

\subsection{Time passage of the buses}

In the following, the departure and arrival times of buses at different stations are presented. For $\mathrm{j}=1$ (first bus), the dates of the first five passages at network stations are given by (equation (6)):

$$
\mathrm{X}^{1}(1)=\left[\begin{array}{c}
e \\
3 \\
26 \\
28 \\
53 \\
54 \\
99
\end{array}\right], \mathrm{X}^{1}(2)=\left[\begin{array}{c}
99 \\
102 \\
125 \\
127 \\
152 \\
153 \\
198
\end{array}\right], \mathrm{X}^{1}(3)=\left[\begin{array}{l}
198 \\
201 \\
224 \\
226 \\
251 \\
252 \\
297
\end{array}\right], \mathrm{X}^{1}(4)=\left[\begin{array}{l}
297 \\
300 \\
323 \\
325 \\
350 \\
351 \\
396
\end{array}\right], \mathrm{X}^{1}(5)=\left[\begin{array}{l}
396 \\
399 \\
422 \\
424 \\
449 \\
450 \\
495
\end{array}\right]
$$

The same calculus is applied for the other bus by replacing $\mathrm{j}$ by 2 in the equation (6). Next, we evaluate boarding, disembarking and waiting time of passengers at every station of the line.

\subsection{Arrival, boarding and waiting time of passengers}

In this section, we are going to evaluate passengers arrival, boarding and waiting time in both departure and connection station (table 5 and 6 ).

The arrival of passengers to their departure station are random according to a exponential distribution of parameter $\lambda=0.7$. Also, the destination of each passenger is given randomly. In the following table, we present passengers arrival in a period where there is not much traffic (off-peak period).

\section{Legend:}

- $\mathrm{k}^{\text {th }}$ (first column) means the $\mathrm{k}^{\text {th }}$ passenger who arrives to this station.

- Arrival time means the arrival of each passenger to the departure station.

- $\mathrm{J}^{\text {th }}$ bus with bus $\mathrm{j}=1,2$ represents the bus that will transport the $\mathrm{k}^{\text {th }}$ passenger (first column).

- $\beta_{s, j}^{i}$ represents the turn of the bus $\mathrm{j}$.

As indicated in table 4 , the departure clearance of bus 1 is given at $t=0$. This bus leaves the departure station at $\mathrm{t}=3 \mathrm{~min}$ (waiting time $=3 \mathrm{~min}$ ). The arrival date of the first passenger is 1,083455 less than 3 , so he can take the bus 1 . The second, third, fourth, fifth and sixth passengers will take also the first bus. However, the seventh one will wait 22,88 min to take the second bus which starts at $t=50 \mathrm{~min}$. At $t=99$, the first bus is back to the starting station and begins a new circuit.

Besides, passengers arrival and waiting times and also buses departure times in a peak period (arrival rate is high compared to the previous table) are presented in figure 11. We notice from this figure that passengers waiting time is very high (average 
Table 5. Passengers boarding and waiting time at the departure station

\begin{tabular}{c||c|c|c|c|c|c|c}
\hline \multicolumn{1}{c||}{} & $\begin{array}{c}\text { Arrival } \\
\text { time(min) }\end{array}$ & Destination & $\begin{array}{c}\mathrm{j}^{\text {th }} \\
\text { bus }\end{array}$ & $\begin{array}{c}\text { Bus } \\
\text { depar- } \\
\text { ture(min) }\end{array}$ & $\beta_{1, j}^{i}$ & $\begin{array}{c}\text { Boarding } \\
\text { time } \\
\mathrm{OM}_{1, j}^{i}(\mathrm{~min})\end{array}$ & $\begin{array}{c}\text { Waiting } \\
\text { time (min) }\end{array}$ \\
\hline 1 & 1,083455 & $\mathrm{~S}_{3}^{i}$ & 1 & 3 & 1 & 1,083455 & 1,92 \\
\hline 2 & 2,123235 & $\mathrm{~S}_{3}^{i+1}$ & 1 & 3 & 1 & 2,123235 & 0,88 \\
\hline 3 & 2,523236 & $\mathrm{~S}_{2}^{i}$ & 1 & 3 & 1 & 2,523236 & 0,48 \\
\hline 4 & 2,632148 & $\mathrm{~S}_{2}^{i}$ & 1 & 3 & 1 & 2,632148 & 0,37 \\
\hline 5 & 2,650012 & $\mathrm{~S}_{3}^{i}$ & 1 & 3 & 1 & 2,732148 & 0,35 \\
\hline 6 & 2,782523 & $\mathrm{~S}_{3}^{i}$ & 1 & 3 & 1 & 2,832148 & 0,22 \\
\hline 7 & 30,12356 & $\mathrm{~S}_{3}^{i+1}$ & 2 & 53 & 1 & 50,1 & 22,88 \\
\hline 8 & 32,20326 & $\mathrm{~S}_{2}^{i}$ & 2 & 53 & 1 & 50,2 & 20,80 \\
\hline 9 & 35,23569 & $\mathrm{~S}_{2}^{i}$ & 2 & 53 & 1 & 50,3 & 17,77 \\
\hline 10 & 50,23546 & $\mathrm{~S}_{2}^{i}$ & 2 & 53 & 1 & 50,4 & 2,77 \\
\hline 11 & 52,23254 & $\mathrm{~S}_{3}^{i}$ & 2 & 53 & 1 & 50,5 & 0,77 \\
\hline 12 & 55,23254 & $\mathrm{~S}_{3}^{i+1}$ & 1 & 102 & 2 & 99,1 & 46,77 \\
\hline 13 & 60,00023 & $\mathrm{~S}_{3}^{i+1}$ & 1 & 102 & 2 & 99,2 & 42 \\
\hline 15 & 65,23564 & $\mathrm{~S}_{3}^{i}$ & 1 & 102 & 2 & 99,3 & 36,77 \\
\hline 16 & 75,25648 & $\mathrm{~S}_{3}^{i}$ & 1 & 102 & 2 & 99,4 & 31,75 \\
\hline
\end{tabular}

of $26 \mathrm{~min})$, because the number of buses used $\left(\mathrm{n}_{i}=2\right)$ is too small for arrival rate of passengers. Later on, the influence of capacity and number of buses on passengers waiting time are presented.

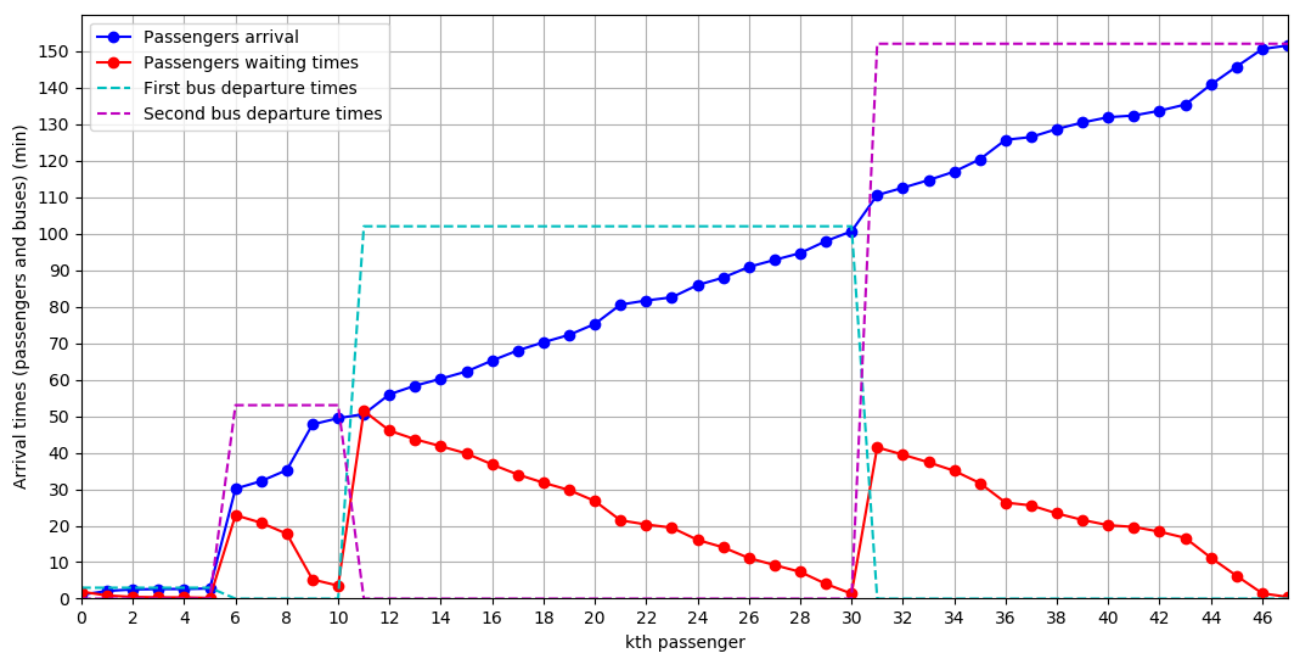

Figure 11. Passengers arrival and waiting time at the departure station

The table 6 describes arrival, boarding and waiting time of passengers at the connection station. The first bus (in its first passage) arrives at the connection station with six passengers on board (see table 5) and finds five waiting passengers. Only three of them get off the bus (passenger 2, 3 and 4 in table 5). We notice that the fourth and fifth passengers of table 6 have made the correspondence. In fact, the first bus of line $\mathrm{L}_{i+1}$ arrives at the connection station at $\mathrm{t}=19$ and puts down two passengers who want 
Table 6. Passengers boarding and waiting time at the connection station

\begin{tabular}{c||c|c|c|c|c|c|c}
\hline \multicolumn{1}{c||}{} & $\begin{array}{c}\text { Arrival } \\
\text { time(min) }\end{array}$ & Destination & $\begin{array}{c}\mathrm{j}^{\text {th }} \\
\text { bus }\end{array}$ & $\begin{array}{c}\text { Bus } \\
\text { depar- } \\
\text { ture(min) }\end{array}$ & $\beta_{2, j}^{i}$ & $\begin{array}{c}\text { Boarding } \\
\text { time } \\
\mathrm{OM}_{2, j}^{i}(\mathrm{~min})\end{array}$ & $\begin{array}{c}\text { Waiting } \\
\text { time (min) }\end{array}$ \\
\hline 1 & 16,02365 & $\mathrm{~S}_{3}^{i}$ & 1 & 28 & 1 & 26,1 & 11,98 \\
\hline 2 & 17,32567 & $\mathrm{~S}_{3}^{i}$ & 1 & 28 & 1 & 26,2 & 10,68 \\
\hline 3 & 18,00035 & $\mathrm{~S}_{3}^{i}$ & 1 & 28 & 1 & 26,3 & 10 \\
\hline 4 & 19,1 & $\mathrm{~S}_{3}^{i}$ & 1 & 28 & 1 & 26,4 & 8,9 \\
\hline 5 & 19,2 & $\mathrm{~S}_{3}^{i}$ & 1 & 28 & 1 & 26,5 & 8,8 \\
\hline 6 & 50,01235 & $\mathrm{~S}_{3}^{i}$ & 2 & 78 & 1 & 76,1 & 27,99 \\
\hline 7 & 52,14589 & $\mathrm{~S}_{3}^{i}$ & 2 & 78 & 1 & 76,2 & 25,86 \\
\hline 8 & 56,14567 & $\mathrm{~S}_{3}^{i}$ & 2 & 78 & 1 & 76,3 & 21,86 \\
\hline 9 & 70,00123 & $\mathrm{~S}_{3}^{i}$ & 2 & 78 & 1 & 76,4 & 8 \\
\hline 10 & 75,23784 & $\mathrm{~S}_{3}^{i}$ & 2 & 78 & 1 & 76,5 & 2,77 \\
\hline
\end{tabular}

to make the correspondence. At $\mathrm{t}=28$, the first bus of line $\mathrm{L}_{i}$ leaves the connection station and goes to the arrival terminus with eight passengers on board. Furthermore, the same approach is applied for the arrival terminus.

\subsection{Passengers disembarking time}

To evaluate disembarking time of passengers at different stations, we use the equation (14) and (15). To illustrate our approach, we are going to calculate disembarking times for passengers who took the first bus (in its first passage) at the departure station (see table 5). In the following table, some routing functions are computed in order to evaluate the daters related to passengers disembarking (see the algorithm in figure 9). We recall that "-" in table below means that the parameter is not computed. The first passenger arrives to the departure station at $\mathrm{t}=1,083455 \mathrm{~min}$ and gets on the first bus $(\mathrm{j}=1)$. The destination of this passenger is station three (destination is $\mathrm{S}_{3}^{i}$ as given in table 7), so the transition $x_{3,1}^{i}$ will be fired. Using the equation (13), we have:

- $\mathrm{x}_{3,1}^{i}(1)=\mathrm{OM}_{1,1}^{i}\left(\xi_{7,1}^{i}\right) \oplus \mathrm{OM}_{2,1}^{i}\left(\xi_{8,1}^{i}\right) \oplus \mathrm{OM}_{3,1}^{i}\left(\xi_{9,1}^{i}\right)=\mathrm{OM}_{1,1}^{i}(1)=1,083455 \mathrm{~min}$.

Firing times of the other transitions are deduced in the same way using the equations (14) and (15) and the algorithm of figure 9 to compute the routing functions. Furthermore, the first bus arrives at $\mathrm{t}=26 \mathrm{~min}$ to the connection station and puts down three passengers. As a result, disembarking times of these passengers are 26,1 min, 26,2 min and 26,3 min. The second passenger want to make correspondence, so the travel time calculated in the bottom of table 7 is just his traveling time on line $\mathrm{L}_{i}$. After getting off, this passenger waits for a bus of line $\mathrm{L}_{i+1}$ to make a correspondence and travel to station three of line $\mathrm{L}_{i+1}$.

\subsection{Influence of buses capacity on passengers waiting times}

In order to show the influence of buses capacity on passengers waiting times, we simulate the evolution of waiting times at the connection stop for many capacity values of circulating buses on the line $\mathrm{L}_{i}$. For instance, we consider mini bus with $\mathrm{C}_{1}^{i}$ $=7$ seats instead of a big bus with 40 seats (figure 12 ). The first bus $(\mathrm{j}=1)$ arrives at the connection station with six passengers on board and finds five waiting passengers. 
Table 7. Passengers traveling time on line $\mathrm{L}_{i}$

\begin{tabular}{c||c|c|c|c|c|c}
\hline $\mathrm{k}^{t h}$ & 1 & 2 & 3 & 4 & 5 & 6 \\
\hline $\mathrm{OM}_{1,1}^{i}$ & 1,083455 & 2,123235 & 2,523236 & 2,632148 & 2,732148 & 2,832148 \\
\hline Destination & $\mathrm{S}_{3}^{i}$ & $\mathrm{~S}_{3}^{i+1}$ & $\mathrm{~S}_{2}^{i}$ & $\mathrm{~S}_{2}^{i}$ & $\mathrm{~S}_{3}^{i}$ & $\mathrm{~S}_{3}^{i}$ \\
\hline$\xi_{4,1}^{i}$ & - & 2 & 3 & 4 & - & - \\
\hline$\xi_{5,1}^{i}$ & - & $\varepsilon$ & $\varepsilon$ & $\varepsilon$ & - & - \\
\hline$\xi_{6,1}^{i}$ & - & $\varepsilon$ & $\varepsilon$ & $\varepsilon$ & - & - \\
\hline$\xi_{7,1}^{i}$ & 1 & - & - & - & 5 & 6 \\
\hline$\xi_{8,1}^{i}$ & $\varepsilon$ & - & - & - & $\varepsilon$ & $\varepsilon$ \\
\hline$\xi_{9,1}^{i}$ & $\varepsilon$ & - & - & - & $\varepsilon$ & $\varepsilon$ \\
\hline $\mathrm{x}_{2,1}^{i}$ & - & 2,123235 & 2,523236 & 2,632148 & - & - \\
\hline $\mathrm{x}_{3,1}^{i}$ & 1,083455 & - & - & - & 2,732148 & 2,832148 \\
\hline$\xi_{11,1}^{i}$ & - & - & 1 & 1 & - & - \\
\hline$\xi_{12,1}^{i}$ & 1 & - & - & - & 1 & 1 \\
\hline$\xi_{13,1}^{i}$ & - & 1 & - & - & - & - \\
\hline$\xi_{14,1}^{i}$ & - & - & 2 & 3 & - & - \\
\hline$\xi_{15,1}^{i}$ & - & 1 & - & - & - & - \\
\hline$\xi_{16,1}^{i}$ & - & - & 1 & 1 & - & - \\
\hline$\xi_{17,1}^{i}$ & - & $\varepsilon$ & - & - & - & - \\
\hline $\mathrm{D}_{1,1}^{i}$ & - & - & - & - & - & - \\
\hline $\mathrm{D}_{2,1}^{i}$ & - & - & 26,2 & 26,3 & - & - \\
\hline $\mathrm{D}_{3,1}^{i}$ & 53,1 & - & - & - & 53,2 & 53,3 \\
\hline $\mathrm{Crp}_{1}^{i}$ & - & 26,1 & - & - & - & - \\
\hline $\operatorname{Travel} \mathrm{time}(\min )$ & 52,016545 & 23.976765 & 23,676764 & 23,667852 & 50.549988 & 50,517477 \\
\hline & & & & & &
\end{tabular}

Only three passengers get off the bus at this station. Since the capacity of the bus does not exceed 7, only four among the waiting passengers can get on the bus. As a result, the fifth passenger waits $58.8 \mathrm{~min}$ instead of $8.8 \mathrm{~min}$ (figure 12). So, the average of passengers waiting time increases. Moreover, in figure 12, we notice that passengers, who have too high peaks $\left(5^{t h}, 18^{\text {th }}, 29^{\text {th }} \ldots\right.$ ), find the bus full and saturated (no empty seats inside). For that reason, they have to wait getting on board the next bus. As a result, their waiting times increases by 50 minutes representing the passage frequency of buses at the considered station.

Furthermore, we can observe on the figure 13 for peak periods scenarios that the two buses could become saturated. Therefore, the average of passengers waiting time increases. These observations allow concluding that this type of buses are not adapted to peak periods. So, in order to eliminate the influence of the limited capacity on passengers waiting time, we calculate the optimal capacity.

\subsection{Optimal capacity}

In this regulation study we consider both criteria that influence passengers waiting time: the capacity of buses and their number on each network line. So, the figure 14 describes the influence of these criteria on passengers waiting time. Our objective to find a compromise between the capacity of buses to use on the line, their number and an acceptable waiting time for passengers. We notice in figure 14 that when we increase the capacity of the bus or number of used buses, passengers waiting time decreases 


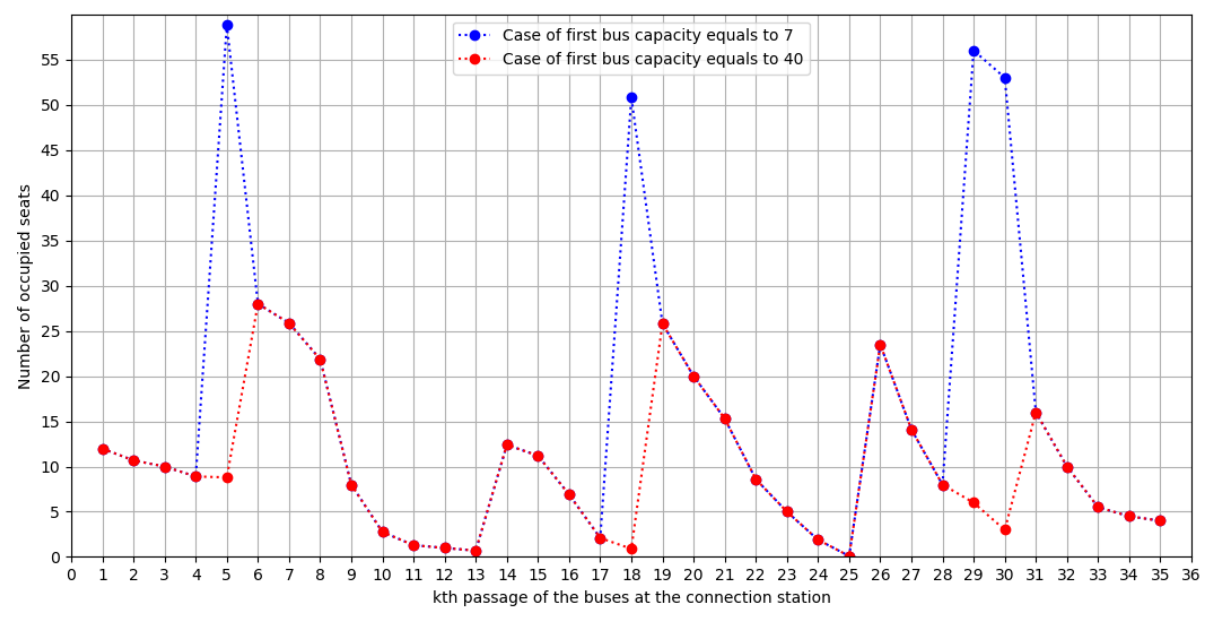

Figure 12. The influence of capacity on passengers waiting time

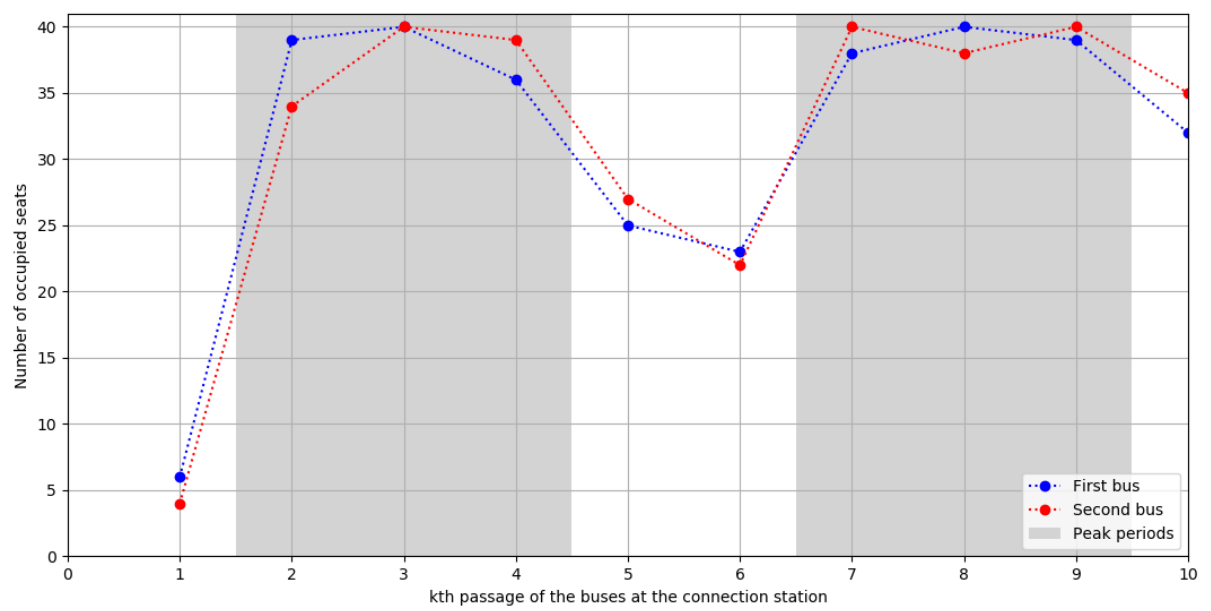

Figure 13. Number of buses occupied seats in a given period

to reach a stable point from which the capacity has no longer impact on passengers waiting time. So, to eliminate the influence of capacity on passengers waiting time, we calculate the parameter $\mathrm{Copt}_{j}^{i}$ (optimal capacity) presented in equation (20). For instance, we notice in figure 14 using only one bus with a capacity $\operatorname{Copt}_{1}^{i}=45$ the average waiting time becomes stable at $17 \mathrm{~min}$. So, to minimize transportation costs, we have to use a bus of 45 seats rather then a bus of seats higher than 45 . On the other hand, it is better to use only one bus with 45 seats with a maximum of $17 \mathrm{~min}$ as waiting time (for some passengers) rather then two buses with 30 seats and an average waiting time reaching $13 \mathrm{~min}$. The figure 14 shows the result of other scenarios using more than two buses and associated average waiting times. 


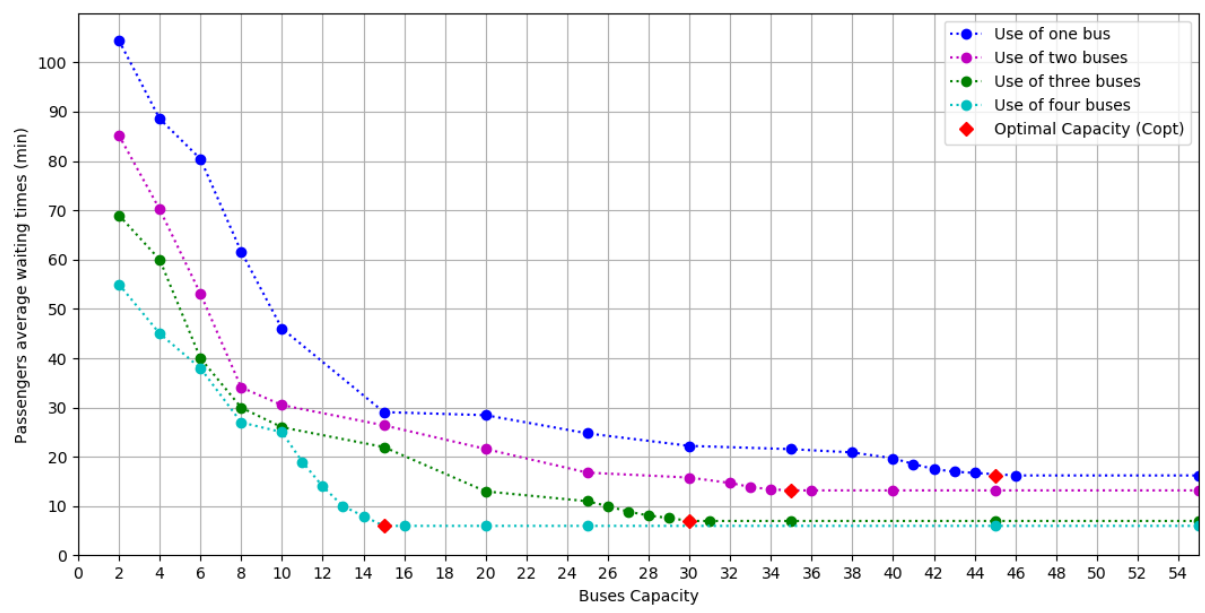

Figure 14. The influence of capacity and number of buses on passengers waiting time

\section{Conclusion and perspectives}

In this study, we proposed a modeling approach using the two complementary tools Petri nets and $(\max ,+)$ algebra to describe the behavior of a bus network with the aim to evaluate, analyze and control its performances. The studied system is subject to complex phenomena such as conflicts and choice situation, synchronization and concurrency. Using $(\max ,+)$ algebra for modeling such phenomena is not obvious. Our objective through this paper is to bring our contribution by proposing a $(\max ,+)$ modeling approach for this type of DES with conflicts and enrich the current emergent theory in this research field. A non stationary $(\max ,+)$ model is then proposed in this paper and routing functions enabling to manage all encountered conflicts in the system are explicitly developed with mathematical equations.

Simulations are performed and obtained results are reported and analyzed. Firstly, we evaluate some system metrics such as arrival and departure time of buses at different stations of the network and also waiting, boarding, disembarking times of each passenger. Furthermore, we study the influence of limited capacity of buses on passengers waiting time. Then, we propose a control approach enabling to determine a compromise between the capacity and number of buses to use on the network in order to minimize both waiting times of passengers and consequently engaged transportation company costs. A mathematical expression of optimal capacity is proposed and illustrated. Obtained results are promising.

Although Petri nets provide a good paradigm for modeling transportation networks, there are some improvements to be addressed as a future work. Modeling large networks with more details could conduct to a combinatorial problem. Our perspectives can then include the use of Colored Petri Nets (CPN) in order to reduce and simplify the proposed graphical model. We intend also to develop and extend the $(\max ,+)$ theory for translating $\mathrm{CPN}$ models to $(\max ,+)$ equations. Moreover, some theories in $(\max ,+)$ algebra can be developed to improve the quality of services offered to passengers in terms of traveling and waiting times. This improvement will also be benefit to the transportation companies while reducing their costs and environmental impact (gas emissions) by minimizing the number of buses to deploy on the network. Furthermore, we intend to generalize in our future models the stochastic aspect of 
the system by integrating other stochastic parameters such as buses travel times often variable.

\section{References}

Adler, Jeffrey L, and Victor J Blue. 2002. "A cooperative multi-agent transportation management and route guidance system." Transportation Research Part C: Emerging Technologies 10:433-454.

Baccelli, Francois, Guy Cohen, Geert Jan Olsder, and Jean-Pierre Quadrat. 1992. Synchronization and linearity: An Algebra for Discrete Event Systems. New York: J. Wiley.

Baccelli, Francois, S. Foss, and Bruno Gaujal. 1996. "Free-choice Petri nets-an algebraic approach." IEEE Transactions on Automatic Control 41 (12): 1751 - 1778.

Benarbia, Taha, Karim Labadi, Darcherif Abdel Moumen, and Maurice Chayet. 2012. "Modelling and control of self-Service Public Bicycle Systems by using Petri Nets." International Journal of Modeling, Identification and control (IJMIC) 17 (3): 173-194.

Bonhomme, Patrice. 2013. "Scheduling and control of real-time systems based on a token player approach." Discrete Event Dynamic Systems Theory and Applications, Springer 23 (2): 191-209.

Borges, Barbosa Samuel, Marcelo Gitirana Gomes Ferreira, Nickel Elton Moura, Cruz Jorge Alcides, Forcellini Fernando Antnio, Garcia Jssica, and Guerra Jos Baltazar Salgueirinho Osrio de Andrade. 2016. "Multi-criteria analysis model to evaluate transport systems: An application in Florianpolis, Brazil." Transportation Research Part A 96:1-13.

Boussahel, Wassim Mohamed, Said Amari, and Redouane Kara. 2016. "Analytic evaluation of the cycle time on networked conflicting timed event graphs in the (Max, + ) algebra." Discrete Event Dynamic Systems Theory and Applications, Springer 26 (4): 561-581.

Bouyekhf, Rachid, Abdeljalil Abbas-Turki, O Grunder, and Abdallah El Moudni. 2003. "Modelling, performance evaluation and planning of public transport systems using generalized stochastic Petri nets." Transport Reviews: A Transnational Transdisciplinary Journal 23:1, 51-69.

Cacchiani, Valentina, Dennis Huisman, Martin Kidd, Leo Kroon, Paolo Toth, Lucas Veelenturf, and Joris Wagenaar. 2014. "An overview of recovery models and algorithms for real-time railway rescheduling." Transportation Research Part B: Methodological, Elsevier 63: 15-37.

Capkovic, Frantisek. 2016. "Agent-Based Approach to Modelling, Analysing and Performance Evaluation of Discret-Event Systems." IEEE 8th International Conference on Intelligent Systems .

Diaz, M. 2001. "Les réseaux de Petri Modèles fondamentaux." Herms science publications .

Dridi, Mahjoub. 2004. "Contribution à la résolution des problèmes de régulation dans les systèmes de transport dans un contexte multicritère par approche évolutionniste." $\mathrm{PhD}$ diss., UST de Lille et de EC de Lille.

Gaubert, Stephane. 1999. "Systemes Dynamiques à événements discrets." Lecture notes, ENSMP, Automation and Master degree ATS Orsay, Inria, Rocquencourt, France .

Hakim, Laichour. 2002. "Modélisation multi-agent et aide à la décision : application à la régulation des correspondances dans les réseaux de transport urbain." PhD diss., UST de Lille.

Heidergott, B., and R. de Vries. 2001. "Towards a (Max,+) Control Theory for Public Transportation Networks." Discrete Event Dynamic Systems Theory and Applications, Springer 11 (4): 371-398.

Houssin, Laurent, Sébastien Lahaye, and Jean-Luis Boimond. 2006. "Timetable synthesis using (max,+) algebra." IFAC Proceedings Volumes 39 (3): 375-380.

Houssin, Laurent, Sébastien Lahaye, and Jean-Luis Boimond. 2013. "Control of (max,+) algebra linear systems minimizing delays." Discrete Event Dynamic Systems 23 (3): 261-276.

Idel Mahjoub, Yassine, Elhoucine Chakir El Alaoui, and Ahmed Nait-Sidi-Moh. 2017. "Mod- 
eling and Performance Evaluation of a Public Transportation System." In proceedings of IEEE, Systems, Signals and Devices (SSD) 407-412.

Kersbergen, Bart, Jànos Rudan, Ton van den Boom, and Bart De Schutter. 2016. "Towards railway traffic management using switching Max-plus-linear systems." Discrete Event Dynamic Systems Theory and Applications, Springer 26 (2): 183-223.

Labadi, Karim, Taha Benarbia, Jean-Pierre Barbot, Samir Hamaci, and Abdelhafid Omari. 2015. "Stochastic Petri Net Modeling, Simulation and Analysis of Public Bicycle Sharing Systems." IEEE Transactions on Automation Science and Engineering 12 (4): 1380 - 1395.

Lahaye, Sébastien, Laurent Houssin, and Jean-Luis Boimond. 2003. "Modelling of urban bus networks in dioids algebra." Positive Systems 294: 23-30.

List, George, and Mehdi Mashayekhi. 2015. "A Modular Colored Stochastic Petri Net for Modeling and Analysis of Signalized Intersections." IEEE Transaction on intellegent transportation systems 17 (3): $701-713$.

Murata, T. 1989. "Petri nets: Properties, analysis and applications." Proceedings of the IEEE 77 (4): $541-580$.

Nait-Sidi-Moh, Ahmed, Marie-Ange Manier, and Abdellah El Moudni. 2003. "Performance Analysis of a Bus Network Based on Petri Nets and (Max,+) Algebra." Systems Analysis Modelling Simulation (SAMS) 43 (5): 639-669.

Nait-Sidi-Moh, Ahmed, Marie-Ange Manier, and Abdellah El Moudni. 2009. "Spectral analysis for performance evaluation in a bus network." In european journal of operational research 193 (1): 289-302.

Nielsen, Bo Friis, Laura Frolich, Otto Anker Nielsen, and Dorte Filges. 2014. "Estimating passenger numbers in trains using existing weighing capabilities." Transportmetrica A: Transport Science 502-517.

Nuzzolo, Agostino, and Antonio Comi. 2016. "Advanced public transport and intelligent transport systems: new modelling challenges." Transportmetrica A: Transport Science .

Nuzzolo, Agostino, and Antonio Comi. 2017. "Transit travel strategy as solution of a Markov decision problem: Theory and applications." In 5th IEEE International Conference on Models and Technologies for Intelligent Transportation Systems pp. 850-855.

Ould-Sidi, Mohamed Mahmoud, Said Hayat, Slim Hammadi, and Pierre Borne. 2006. "Urban transport networks regulation and evaluation : A fuzzy evolutionary approach." IEEE Transaction on Systems Mans and Cybernetics .

Reisig, Wolfgang, and Grzegorz Rozenberg. 1998. Lectures on Petri Nets I: Basic Models: Advances in Petri Nets. Springer.

Yeh, Chung-Hsing, Hepu Deng, and Yu-Hern Chang. 2000. "Fuzzy multicriteria analysis for performance evaluation of bus companies." European Journal of Operational Research 129:459-473. 